\title{
Money, Capital and Exchange Rate Fluctuations*
}

\author{
Pere Gomis-Porqueras, Timothy Kam, and Junsang Lee ${ }^{\dagger}$
}

March 2, 2012

\begin{abstract}
We explore how the informational frictions underlying monetary exchange affect international exchange rate dynamics. Our perfectly flexible price model is capable of producing endogenously rigid international relative prices in response to technology and monetary shocks. The model is capable of accounting for the empirical regularities that the real and nominal exchange rates are more volatile than U.S. output, and that the two are positively and perfectly correlated. The model is also consistent with other standard real business cycle facts for the U.S.
\end{abstract}

JEL Classification: E31; E32; E43; E44

Keywords: Search; Money; Open Economy; Exchange Rate Puzzle

\section{Introduction}

It is well known that the real and nominal exchange rates of the world's largest economies are very volatile and persistent. Moreover, these two time series are perfectly and positively correlated. The seminal work of Chari et al. (2002) explored whether these empirical regularities could be understood in the context of a standard two-country business cycle model with sticky prices. They concluded that such models can account for the volatility of the exchange rates, but not their persistence. Sticky price models are able to generate volatile real and nominal

*First submitted: November 16, 2010; Revised and Resubmitted: November 7, 2011; Accepted: January 25, 2012.

${ }^{\dagger}$ We would like to thank the editor Harold Cole, and two anonymous referees for valuable comments and suggestions that have significantly improved the paper. T. Kam and J. Lee would like to thank Benoît Julien and Randy Wright for insightful discussions on initial ideas. We also thank Dean Corbae, Chris Edmond, Ian King and seminar participants at the University of Melbourne, Deakin, Macquarie, National Taiwan University, the St. Louis Fed, Bank of Japan, Korea Development Institute, University of Tokyo, and the Department of Economics at Hitotsubashi University for suggestions. Many thanks also go to Chris Waller, David Andolfatto, Julen Esteban-Pretel, Carlos Garriga, Finn Kydland, Adrian Peralta-Alva, Kosuke Aoki, Yukinobu Kitamura, Etsuro Shioji, Nao Sudo, for comments and extended discussions. We would also like to thank the 2010 Chicago Fed Money Workshop participants and especially Yiting Li and Guillaume Rocheteau for their valuable suggestions. T. Kam would also like to acknowledge funding support from the ANU CBE CIGS R62888CG88 and the ARC Discovery Project DP0984399. Correspondence: timothy.kam@anu.edu.au (T. Kam) or junsang@kdi.re.kr (J. Lee). 
exchange rate processes, because, by assumption prices are made to not adjust too quickly to aggregate shocks. In an open economy, the nominal exchange rate and therefore, the real exchange rate, have to overreact. This is a manifestation of the textbook Dornbusch (1976) exchange rate overshooting hypothesis.

In this paper, we examine whether a flexible price, two-country, search theoretic model of money is able to account for the empirical regularities observed in U.S. real and nominal exchange rates. ${ }^{1}$ We consider a two-country stochastic version of Lagos and Wright (2005) and Aruoba et al. (2011), where there exist two sectors or sequential submarkets within each period. These sectors comprise a decentralized market (DM) with anonymous (or partially anonymous) trading, and, a centralized Walrasian market (CM). We assume that international trade and asset flows occur in the model's frictionless CM. The CM assumption allows direct comparisons with existing international monetary business cycle models with flexible prices (e.g. Schlagenhauf and Wrase, 1995) and models with sticky prices (e.g. Chari et al., 2002), while providing a deeper foundation for domestic money and an alternative equilibrium restriction on pricing processes. Following Aruoba et al. (2011), we allow for installed capital in each centralized market $(\mathrm{CM})$ to be a productive input for sellers in each subsequent decentralized market (DM). This aspect of "capital complementarity" generates an equilibrium linkage between inflation and real economic activity across the DM and CM.

There are two key mechanisms at work in this model which help amplify and propagate international business cycle shocks. We label them "anonymity" and "DM capital complementarity". The first mechanism is anonymity. Anonymity is a term for: (i) The lack of, or imperfect, record-keeping of individual trader's histories; (ii) Nonexistence of public communication of individual trading histories; and (iii) Lack of enforcement of private contracts. Given this assumption of anonymity and coupled with a random market participation (or meeting) environment (which gives rise to a lack of double coincidence of wants), the domestic currency has indirect value as a medium of exchange and store of value (i.e. serves a precautionary asset

\footnotetext{
${ }^{1}$ Alessandria (2009) also departs from the standard Walrasian business cycle framework. He develops a model where in each country, there is a "large family" consisting of a continuum of worker-shoppers who engage in noisy search (i.e. the number of price quotes each shopper faces is a random variable) $\grave{a}$ la Burdett and Judd (1983). The shoppers aim to find the "best" price of a single unit of a good offered by domestic or foreign firms. The opportunity cost of search is a function of the worker-shopper's forgone real wage. Because of shoppers' objective to find the best quote and such search is noisy, firms can price discriminate across markets. The equilibrium distributions of prices will be different across countries as a function of international relative real wages. Given relative aggregate country-specific technology and/or taste shocks, which change cross-country relative real wages, the distribution of prices in the home country will shift relative to that in the foreign country. This results in an endogenous deviation from the law of one price, and hence large cross-country relative price fluctuations at the both the aggregate and disaggregated levels.

In contrast to Alessandria (2009), our key friction is a monetary one and arises only in a specific decentralized sector of each country. There is no cross-country search by buyers in our model. Our centralized market (CM), where international trade and asset flows determine the nominal and real exchange rates, is similar to standard Walrasian international business cycle models. This feature facilitates closer comparison with existing international monetary models (e.g. Chari et al., 2002; Schlagenhauf and Wrase, 1995). Moreover, given that we have a monetary model, we can also have something to say about the empirical regularity that the international real and nominal exchange rates for the U.S. are perfectly and positively correlated.
} 
function). This friction induces asset market incompleteness in the sense that individuals are unable to fully insure against their stochastic trading opportunities in the DM.

In our benchmark model with logarithmic utility functions and DM price taking, we can contrast our DM equilibrium pricing condition with a standard model's cash-in-advance (CIA) constraint. In particular, the CIA constraint appears as a reduced-form special case of our equilibrium condition. Since our DM equilibrium pricing condition relates to buyers' and sellers' primitive preferences and technologies, then, money supply and technology shocks become directly encoded in the DM equilibrium pricing condition. Depending on the DM Walrasian pricing protocol (or sharing rule in a bargaining version), domestic prices need not respond by as much to home technology and money supply growth shocks. This would also be true in the foreign country. Thus in the equilibrium of our calibrated model, we show that relative aggregate prices across countries do not respond as much to country-specific technology or money supply growth shocks. This explains why the model is able to account for the volatility of the exchange rates. We also show that anonymity also accounts for some persistence in relative prices and hence international exchange rates.

The second mechanism in our model is DM capital complementarity. Capital complementarity provides for an additional return on capital which places additional restriction on the equilibrium asset pricing relations for money and capital. We also show that this feature helps to account for excess volatility and persistence in equilibrium relative prices and international exchange rates.

In our calibrated experiments, we show that, individually, anonymity and capital complementarity in sellers' production technology in the DM can account for the observed excess volatility in the exchange rates. Moreover, in the benchmark calibrated model, where both features are present, we can account even better for this empirical regularity.

In our model, the assumption of (some) anonymous trades in the DM is intertwined with the DM as a non-traded goods sector. To disentangle the contribution of anonymity and the role of the non-tradable sector on the exchange rate dynamics, we relax the anonymity assumption, as in Aruoba et al. (2011). In particular, we introduce an exogenous probability that agents in each DM may be segmented into one of two kinds of trades: anonymous monetary trades or monitored trades which allow for exchange with credit. By considering the limit of pure credit trades in the DM, we are able to shut down the role of monetary friction and isolate the effect on exchange rate dynamics due to the non-monetary DM as a non-traded goods sector. We show that the latter alone cannot account for the stylized facts on the real exchange rate for the U.S. However, in the presence of a small degree of anonymity in the DM, cross-country aggregate relative prices are non-volatile and persistent, in response to aggregate technology and money supply growth shocks. This contributes to the excess volatility and persistence in the real and nominal exchange rates. Also, without requiring exogenous price-stickiness (e.g. 
Chari et al., 2002) nor additional shocks (e.g. Steinsson, 2008), the benchmark model is also able to rationalize near perfect positive correlation between the real and nominal exchange rate.

The paper is organized as follows. In section 2, we outline the details and assumption of the baseline quantitative-theoretical model. We then work through the model's stationary Markov monetary equilibrium and its implications in Section 3. Next, in Section 4, we provide some insight into the key mechanisms in the model, and explain the potential trade-offs and the role of the DM pricing protocol in accounting for relative pricing and exchange rate behavior. We then take the theory to the data in Section 5. We discuss the model's business cycle features relative to the data and other existing models in Section 6. We then verify how the mechanisms interact to produce the business cycle features, by isolating each mechanism, in Section 6.1. We conclude in Section 7.

\section{Environment}

Consider a two-country model, each referred to as Home and Foreign. Variables and parameters without an asterisk (or with a subscript $h$ ) will refer to the Home country, and those with an asterisk (or with a subscript $f$ ), will refer to the Foreign country. Time is denumerable, and a time period is denoted by $t \in \mathbb{N}:=\{0,1,2, \ldots\}$. Agents exist on a continuum $[0,1]$ and have a common discount factor $\beta \in(0,1)$. Each $t \in \mathbb{N}$ is composed of two arbitrary sub-periods, night and day. At night, agents trade anonymously in decentralized markets (DM). During the day, agents trade in Walrasian centralized markets (CM). The nature of consumption, production and trade in each market will be explained in detail in sections 2.7 and 2.8 .

\subsection{Preferences and DM technology}

Denote $q^{b} \in \mathbb{R}_{+}$as an agent's consumption (as a buyer) and $q^{s} \in \mathbb{R}_{+}$as an agent's output (as a seller) of a "specialized", or, agent-specific and non-storable good in the DM. Similar to Lagos and Wright (2005), each agent can be a producer of a special $q^{s}$, and is assumed to not value his own product. Let $X \in \mathbb{R}_{+}, k \in \mathbb{R}_{+}$and $H \in[0, \bar{H}]$, where $\bar{H}<+\infty$, denote consumption of a general good in the CM, individual capital stock and labor in the CM, respectively. Agents' perperiod preferences are represented by $\left(q^{b}, q^{s}, X, H, z\right) \mapsto u\left(q^{b}\right)-c\left(q^{s} / z, k\right)+U(X)-h(H)$, where $u(q)$ is the per-period payoff from consuming $q, z$ is aggregate home total factor productivity, and $c(q / z, k)$ is the utility cost of producing $q$ with fixed within-period capital, $k$, determined in the previous $\mathrm{CM}^{2} U(X)$ is the immediate payoff from consuming $X$ in the CM, and $-h(H)$

\footnotetext{
${ }^{2}$ Or equivalently, let $H_{D M_{\tilde{F}}}$ be the labor effort of an agent expended in a DM. Suppose the production technology, $\left(H_{D M}, k, z\right) \mapsto z \cdot \tilde{F}\left(H_{D M}, k\right)$ using capital and labor, is bijective and homogeneous of degree one. Then $H_{D M}=\tilde{F} \tilde{F}^{-1}\left(q^{s} / z k\right) \cdot k$ and $c\left(q^{s} / z, k\right) \equiv H_{D M}$. Our quantitative exercise will use a Cobb-Douglas example for $\tilde{F}(\cdot, \cdot ; \varpi)$ where $1 / \varpi \in(0,1)$ is the labor share.
} 
is the disutility of work effort in the CM. We make the following assumptions.

Assumption 1 The functions $u, U, h: \mathbb{R}_{+} \rightarrow \mathbb{R}$ and $c: \mathbb{R}_{+}^{2} \rightarrow \mathbb{R}$ have the following properties:

(i) First and second derivatives exist everywhere: $u, U \in \mathbf{C}^{2}\left(\mathbb{R}_{+}\right)$and $c \in \mathbf{C}^{2}\left(\mathbb{R}_{+}^{2}\right)$;

(ii) $u_{q}>0, c_{q}>0, c_{k}<0, U_{X}>0, h_{H}>0$ and constant;

(iii) $u_{q q}<0, c_{q q} \geq 0, c_{q k}<0, U_{X X} \leq 0$ and $h_{H H}=0$;

(iv) $u(0)=c(0,0)=0$; and

(v) $u(q)>c(q / z, k)$ for every $(q / z, k)$.

\subsection{DM access (or matching) technology}

In our benchmark economy with DM competitive price taking, we assume that there is a probability $\sigma \leq 1 / 2$ that each agent can access the DM as a buyer. With symmetric probability $\sigma$, the agent can access the DM to sell his special good. With probability $1-2 \sigma$, an agent cannot access the DM, or equivalently, will leave the DM with no exchange. ${ }^{3}$ For simplicity, assume that "double-coincidence-of-wants" events (where buyers and sellers in the DM are able to barter), and, the event where an agent can simultaneously buy $q^{b}$ and sell $q^{s}$, occur with probability zero.

\subsection{CM technology}

In the CM the final good in the Home country is produced according to a constant returns technology, $\left(y_{h}, y_{f}\right) \mapsto G\left(y_{h}, y_{f}\right)$, where $y_{h}$ denotes the input demand for an intermediate good produced in the home country, and, $y_{f}$ represents the demand of a substitutable input produced in the foreign country. Assume that $G \in \mathbf{C}^{2}\left(\mathbb{R}_{+}^{2}\right), G_{i}>0, G_{j}>0, G_{i i}<0$, and $G_{j j}<0$, where $i, j \in\left\{y_{h}, y_{f}\right\}$. Similarly, the foreign final good production function is given by, $\left(y_{f}^{*}, y_{h}^{*}\right) \mapsto G\left(y_{f}^{*}, y_{h}^{*}\right)$.

Let $K$ denote an aggregate capital stock in each home country. The production of the different intermediate goods are given by another constant returns technology, $(K, H) \mapsto z F(K, H)$ which is subject to a stochastic productivity shock, $z$. Assume $\left(z_{t}\right)_{t \in \mathbb{N}}$ is a strictly positive and bounded stochastic process. Assume that $F \in \mathbf{C}^{2}\left(\mathbb{R}_{+}^{2}\right)$ and that $F_{K}>0, F_{H}>0, F_{K K}<$ $0, F_{H H}<0$, and, $F(K, 0)=F(0, H)=0$.

\footnotetext{
${ }^{3}$ As pointed out by Rocheteau and Wright (2005), this "competitive equilibrium" interpretation can be thought as a generalization of Lucas and Prescott (1974) and Alvarez and Veracierto (2000) and is still consistent with the essentiality of money, as long as we maintain anonymity and events with a double-coincidence-of-wants problem. Later on, when we consider DM bargaining (proportional and generalized Nash bargaining) in bilateral matches, the interpretation of $\sigma$ then is that of either the probability that the agent as buyer meets a seller of a special good he wishes to consume, or, the symmetric probability that the same agent, as seller, meets a buyer who wants to buy his product.
} 


\subsection{Assets and individual state variables}

Let $m \in \mathbb{R}_{+}$be the stock of an agent's local nominal money holding in the Home country. Denote $b$ as the current stock of an internationally traded complete state-contingent money claim, held by an agent in the Home country. Each $b$ is denominated in the Home currency. Since these complete contingent claims require knowledge of traders' histories, it is natural that they are not issued or traded in the DM with anonymous randomly matched trades. They are traded only during each CM subperiod. We assume that $k$ cannot be used as a means of payment in the DM since it is not portable. ${ }^{4}$

Now we introduce a modelling device that will help us identify the role of anonymity or monetary friction in the model. Following Aruoba et al. (2011), suppose that conditional on the events of buying, or selling, the exogenous probability that a buyer or seller would engage in an exchange where record keeping is possible is $(1-\kappa) \in[0,1]$. That is, the event that a buyer or a seller can buy or sell a good in the DM using credit occurs with the discrete probability measure $\sigma(1-\kappa)$. Since credit is assumed to be enforceable in such an event, a buyer is willing to take (and a seller is willing to give) out the nominal loan $l$ in exchange for a good, say $\breve{q}$. This loan is required to be repaid in full in the following CM. Then we let $q$ denote a DM specialized good that is exchanged for money in events where exchange occurs with measure $\sigma \kappa$ for a buyer or seller.

Thus we have two distinct markets, one for anonymous traders where cash is needed and one where credit is available. In particular, a fraction $\sigma(1-\kappa)$ of agents can trade in DM with credit, while a fraction $\sigma \kappa$ of agents trade only using fiat money. This is useful because when $\kappa=0$, we are able to shut down the source of monetary friction - the anonymity assumption - and the resulting limit economy is a version of a two-sector real business cycle model with traded and nontraded goods.

Our previous assumptions warrant some remark. ${ }^{5}$ The objective of the paper is not to provide a theory for the coexistence of money and other assets. In our model, we consider the restriction that agents can only use the local currency to buy goods in DM trades where money is needed. If we allow traders in monetary DM exchanges to buy any good with any currency, there will be a problem with the determinacy of the equilibrium composition of currencies. This would then result in an indeterminacy of the nominal exchange rate in equilibrium as well. The restriction to using local currency for local monetary trades is standard in international monetary business cycle models with a domestic currency CIA constraint (e.g. Schlagenhauf and Wrase, 1995). While clearly a deficiency in terms of monetary theory, our assumed restrictions might be rationalized by underlying private information problems in

\footnotetext{
${ }^{4}$ In the DM our agents have their capital physically fixed in place at production sites. Thus, a buyer must visit randomly the location of a seller, and since capital is not portable, it cannot be used for payment, while currency can. This use of spatial separation is in the spirit of the "worker-shopper" idea.

${ }^{5}$ We thank an anonymous referee for making this suggestion.
} 
payment arrangements. In particular, one possible microfoundation for these restrictions on medium of exchange lies in sellers' unwillingness to accept a foreign currency or foreign assets, as a result of private information about the quality of these foreign currencies. These more microfounded justifications are examined by Lester et al. (2008) and Li and Rocheteau (2009). However, these explorations are beyond the scope of this paper.

\subsection{State variables}

Denote the vector of exogenous shocks as $\mathbf{z} \in Z$. We consider Home and Foreign, technology $(z)$ and money supply growth $(\psi)$, shocks. Thus $\mathbf{z}:=\left(z, z^{*}, \psi, \psi^{*}\right)$, and $Z$ is a compact cube in $\mathbb{R}_{+}^{2} \times \mathbb{R}^{2}$. Let the time-t aggregate (global) CM state vector relevant to an agent in country $i \in\{h, f\}$ be denoted by $\mathbf{s}:=\left(M, M^{*}, B, B^{*}, K, K^{*}, \phi, \phi^{*}, e, \mu_{h}, \mu_{f}, \mathbf{z}\right)$. These state variables are defined as follows. The Home aggregate money stock, total private state contingent claims, and capital stock are, respectively, $M, B$ and $K$. The value of money in the Home CM is $\phi:=1 / p_{X}$, where $p_{X}$ is the price level of the Home CM general goods. Similarly, the asterisked variables pertain to the Foreign country's aggregate state variables. The nominal exchange rate in Home CM currency terms is $e$. For country $i, \mu_{i}(\cdot, \mathbf{z}): \mathcal{B}_{i}(\mathbf{z}) \rightarrow[0,1]$ is the time- $t$ probability measure on the Borel $\sigma$-field $\mathcal{B}_{i}(\mathbf{z})$ generated by $(m, b, k, l)$, at each vector of exogenous state variables, $\mathbf{z}^{6}$

At the beginning of the time- $t$ DM, the aggregate (global) state vector for an agent in country $i \in\{h, f\}$ is $\hat{\mathbf{s}}:=\left(M, M^{*}, B, B^{*}, K, K^{*}, \phi, \phi^{*}, e, \nu_{h}, \nu_{f}, \mathbf{z}\right)$. The explicit switch in notation from $\nu_{i}$ to $\mu_{i}$ takes into account that, in general, the distribution of assets upon the economy $i$ entering each period's DM, $\nu_{i}$, may be different from the distribution $\mu_{i}$ upon its leaving the DM, and into the $\mathrm{CM}$, in the same period. ${ }^{7}$

\subsection{Timing}

Figure 1 depicts the sequence of events within each $t \in \mathbb{N}$. The relevant aggregate state vector $\mathbf{s}$ is realized at the beginning of each $t$. This is public information for all agents. An agent in the Home country, first entering the DM with assets $(m, b, k, l)=(m, b, k, 0)$, given $\hat{\mathbf{s}}$, is publicly known by the individual state $(\mathbf{a}, \hat{\mathbf{s}}):=(m, b, k, 0, \hat{\mathbf{s}})$. His indirect utility value of that state is $V(\mathbf{a}, \hat{\mathbf{s}})$. For simplicity, we make the restriction that each country- $i$ agent does not hold another country's currency as an asset. ${ }^{8}$ Since trading opportunities in the DM are random,

\footnotetext{
${ }^{6}$ Note that if $Z=\emptyset$, i.e. in the absence of aggregate exogenous shocks, then the solution of the Markov equilibrium is characterized by a deterministic difference equation system, as in Lagos and Wright (2005). Also, note that the aggregate prices $\left(\phi, \phi^{*}, e\right)$ are explicitly included as (auxiliary) state variables, following Duffie et al. (1994), so that we can restrict our characterization of equilibria to stationary Markov equilibria.

${ }^{7}$ It is straightforward to prove that the probability measures $\nu_{i}$ for each $i \in\{h, f\}$, is degenerate in any equilibrium, as a stochastic extension to the original proof in Lagos and Wright (2004). This affords us plenty of tractability and ease of computation later.

${ }^{8}$ See Head and Shi (2003) for the environment where agents trade currency internationally.
} 
agents within each country $i$ only know the state of their trade partners ex post. Ex ante they only know the probability distribution of traders in the DM, which is $(\sigma, \sigma, 1-2 \sigma)$ with support $\{$ Buyer, Seller, Neither $\}$. Conditional on either events $\{$ Buyer $\}$ or $\{$ Seller $\}$, there is an identical distribution $\{\kappa, 1-\kappa\}$ faced by the agent of a trade being either anonymous (monetary) or monitored (credit).

\section{[ Figure 1 about here. ]}

Upon leaving the DM, an agent's individual state changes to

$$
\left(\mathbf{a}^{\prime}, \mathbf{s}\right):= \begin{cases}\left(m^{\prime}, b, k, 0, \mathbf{s}\right) & \text { w.p. } 2 \sigma \kappa \\ (m, b, k, l, \mathbf{s}) & \text { w.p. } 2 \sigma(1-\kappa)\end{cases}
$$

reflecting the possibility that money had changed hands as a result of the agent being a buyer or seller. As a result of that, the distribution of assets (namely money) would also have changed from $\nu_{i} \in \hat{\mathbf{s}}$ to $\mu_{i} \in \mathbf{s}$. The components $(b, k)$ have not changed since they are predetermined at the beginning of $t$. Thus, within $t$, the agent enters the CM with possible state $\left(\mathbf{a}^{\prime}, \mathbf{s}\right)$ and his value of that state is $W\left(\mathbf{a}^{\prime}, \mathbf{s}\right)$. Agents do not discount payoffs within each period $t$.

Note that there are two key assumptions that yield money supply shocks having real effects: (i) The timing of money supply shocks that must occur at the beginning of each DM; and (ii) the complementarity of capital in the DM. Consider the feature (i). If instead, money supply shocks were to occur at the start of each CM, given completely flexible prices, real quantities and relative prices will not change, as in a typical real-business-cycle model with money. Consequently, as agents enter the subsequent DM with their real money balances, consumption and capital stocks unchanged, the money supply shock at the start of the CM also has no effect on the following DM equilibrium; and in particular, $q$. Now consider feature (ii). This is the assumption of Aruoba et al. (2011) that allows for monetary changes in the DM to affect real allocations in the CM as well. Without it, we have a decoupled dynamic equilibrium in terms of separate CM and DM allocations; i.e. money is neutral (see Aruoba and Wright, 2003).

In the next two sections we describe in detail the sub-period problems, DM and CM, in a backward fashion. To economize on notation, we use the following convention. A variable or vector with a "+" subscript will denote its time $t+1$ contingent outcome. A state with a "_" subscript will denote its time $t-1$ realization. However, in some cases, variables with a "+" subscript, such as money, capital and bonds, are predetermined at the beginning of time $t+1$. In such cases, these are decision or control variables which will be made obvious in the problems below. The same variable without the "+" or "-" subscript denotes its current or time- $t$ realization. 


\subsection{Centralized markets}

In the Home CM, an agent consumes a general good $X \in \mathbb{R}_{+}$which is produced using CMspecific labor $H \in \mathbb{R}_{+}$and capital $k$. In contrast to Lagos and Wright (2005), we introduce a set of internationally traded complete nominal state-contingent claims. Agents in each country's CM who consume more (less) than their total wealth can also trade in these securities.

Let $h(H)=A \cdot H$, where $A>0$ is a constant marginal disutility of work effort. Let $\delta \in[0,1]$ be the depreciation rate of capital and $\tau_{K}$ a proportional tax rate on capital income. Denote $\tilde{r}(\mathbf{s})$ and $\tilde{w}(\mathbf{s})$ as competitive rates of return to capital and labor services, respectively. Then $r:=r(\mathbf{s}) \equiv\left(1-\tau_{K}\right)(\tilde{r}(\mathbf{s})-\delta)$ is the after-tax rate of return to capital, net of depreciation. Similarly, $w(\mathbf{s}):=\left(1-\tau_{H}\right) \tilde{w}(\mathbf{s})$ is the after-tax real wage rate. Denote $\tau_{X}$ as the proportional tax rate on CM consumption $X$. Let $m_{+}:=m(\mathbf{a}, \mathbf{s}), k_{+}:=k(\mathbf{a}, \mathbf{s})$, and $b_{+}:=b(\mathbf{a}, \mathbf{s})$, so that $\mathbf{a}_{+}=\left(m_{+}, b_{+}, k_{+}, 0\right) . Q\left(\mathbf{a}_{+}, \mathbf{s}_{+} \mid \mathbf{a}, \mathbf{s}\right)$ is the domestic price of one unit of the state-contingent claim $b\left(\mathbf{a}_{+}, \mathbf{s}_{+} \mid \mathbf{a}, \mathbf{s}\right)$. Let $\phi:=\phi(\mathbf{s})=1 / p_{X}(\mathbf{s})$ be the inverse of the price of $X$ (i.e. the CM-good value of a unit of Home currency) in the Home country.

At each $t \in \mathbb{N}$, a price-taking agent (at the beginning of the CM sub-period in the Home country) named $(m, b, k, l, \mathbf{s})$ solves the recursive problem given by

$$
\begin{aligned}
W(m, b, k, l, \mathbf{s})=\max _{X, H, m_{+}, k_{+}, b_{+}}\left\{\begin{aligned}
U & X)-A H \\
& \left.+\beta \int V\left(m_{+}, b_{+}, k_{+}, 0, \mathbf{s}_{+}\right) \lambda\left(\mathbf{s}, d \hat{\mathbf{s}}_{+}\right)\right\}
\end{aligned}\right.
\end{aligned}
$$

subject to

$$
\mathbf{s}_{+}=\mathcal{G}\left(\mathbf{s}, \mathbf{v}_{+}\right), \quad \mathbf{v} \stackrel{\text { i.i.d. }}{\sim} \varphi
$$

and,

$$
\begin{aligned}
\left(1+\tau_{X}\right) X(\mathbf{a}, \mathbf{s})+k(\mathbf{a}, \mathbf{s})-k-\phi(\mathbf{s}) b+T(\mathbf{s}) \\
\quad=\phi(\mathbf{s})[m-m(\mathbf{a}, \mathbf{s})-l]+w(\mathbf{s}) H(\mathbf{a}, \mathbf{s})+r(\mathbf{s}) k \\
\quad-\phi(\mathbf{s}) \iint_{\mathbf{s}_{+}, \mathbf{a}_{+}} b\left(\mathbf{a}_{+}, \mathbf{s}_{+} \mid \mathbf{a}, \mathbf{s}\right) Q\left(\mathbf{a}_{+}, \mathbf{s}_{+} \mid \mathbf{a}, \mathbf{s}\right) \mu_{h}\left(\mathbf{s}_{+}, d \mathbf{a}_{+}\right) \lambda\left(\mathbf{s}, d \mathbf{s}_{+}\right)
\end{aligned}
$$

where $\lambda(\mathbf{s}, \cdot)$, for each given $\mathbf{s}$, is induced by $\mathcal{G} \circ \varphi$, and defines an equilibrium product probability measure over Borel-subsets containing $\hat{\mathbf{s}}_{+}$. Constraint (2) describes a transition law, where the mapping $\mathcal{G}=\mathcal{G}_{\{\mathbf{s}\} \backslash\{\mathbf{z}\}} \circ \mathcal{G}_{\{\mathbf{z}\}}$, with component $\mathcal{G}_{\{\mathbf{s}\} \backslash\{\mathbf{z}\}}$ inducing the $\mathbf{z}$-dependent stochastic process for endogenous aggregate states, $\{\mathbf{s}\} \backslash\{\mathbf{z}\}$, is to be pinned down in equilibrium, and $\left(\mathbf{z}, \mathbf{v}_{+}\right) \mapsto \mathcal{G}_{\{\mathbf{z}\}}\left(\mathbf{z}, \mathbf{v}_{+}\right)$is an exogenous map for the aggregate shocks. Implicit in constraint (2) is the equilibrium transition of the distribution of individual states from the period- $t \mathrm{CM}$, to 
the period- $(t+1) \mathrm{DM}$,

$$
\nu_{h}\left(\hat{\mathbf{s}}_{+}, \cdot\right)=\mathcal{G}_{\nu}\left[\mu_{h}(\mathbf{s}, \cdot), \mathbf{z}_{+}\right]
$$

such that the relevant conditional distribution of assets at the beginning of the time- $(t+1)$ CM subperiod is given by

$$
\mu_{h}\left(\mathbf{s}_{+}, \cdot\right)=\mathcal{G}_{\mu}\left[\nu_{h}\left(\hat{\mathbf{s}}_{+}, \cdot\right), \mathbf{z}_{+}\right] \equiv \mathcal{G}_{\mu} \circ \mathcal{G}_{\nu}\left(\mathbf{s}, \mathbf{z}_{+}\right),
$$

where $\mathcal{G}_{\mu}$ and $\mathcal{G}_{\nu}$ are components of $\mathcal{G}_{\{\mathbf{s}\} \backslash\{\mathbf{z}\}}$.

The sequential state-contingent one-period budget constraint given by (3) says the following. For each given state $(m, b, k, l, \mathbf{s})$, taxable consumption of the general good $X$ is to be financed by the change in real money holdings, by after-tax real labor income $w H$, after-tax real capital income $r k$, net of investment flows to physical capital made in the CM, net of contingent claims in real terms, and net of lump-sum government taxes, $T$.

\subsubsection{Optimal individuals' decisions in the CM}

Eliminating $H$ in (1), using the budget constraint (3), the optimal decision rules satisfy the following conditions for every state $(\mathbf{a}, \mathbf{s})$ and every measurable event containing the continuation state $\left(\mathbf{a}_{+}, \hat{\mathbf{s}}_{+}\right)$.

The optimal trade-off between current CM consumption $X$ and leisure $-H$, given the after-tax real wage $w:=w(\mathbf{s})$, is

$$
X: \quad U_{X}[X(\mathbf{a}, \mathbf{s})]=\frac{A\left(1+\tau_{X}\right)}{w(\mathbf{s})} .
$$

The optimal trade-off between a current increase in marginal utility of $X$ in the CM and the present-value expected marginal value of entering the next-period DM with a marginal increment of money holdings is

$$
m_{+}: \quad \frac{A \phi(\mathbf{s})}{w(\mathbf{s})}=\beta \int V_{m_{+}}\left(m_{+}, b_{+}, k_{+}, 0, \hat{\mathbf{s}}_{+}\right) \lambda\left(\mathbf{s}, d \hat{\mathbf{s}}_{+}\right) .
$$

Similar to condition (7), conditions (8)-(9) below provide the optimal trade-offs between the current utility of consumption of $X$ and the expected discounted marginal value of entering the DM with more assets. Specifically, the optimal choice of the complete state-contingent money claims, or bonds, is given by

$$
\begin{array}{r}
b_{+}(\cdot ; \mathbf{s}): \quad \frac{A \phi(\mathbf{s})}{w(\mathbf{s})}\left[Q\left(\mathbf{a}_{+}, \mathbf{s}_{+} \mid \mathbf{a}, \mathbf{s}\right) \mu_{h}\left(\mathbf{s}_{+}, d \mathbf{a}_{+}\right)\right] \lambda\left(\mathbf{s}, d \hat{\mathbf{s}}_{+}\right) \\
=\beta V_{b_{+}}\left(m_{+}, b_{+}, k_{+}, 0, \hat{\mathbf{s}}_{+}\right),
\end{array}
$$


which holds for every $\mathbf{s}$, every $\hat{\mathbf{s}}_{+}$, and implicitly, every $\mathbf{s}_{+}$.

The optimal choice of the Home-produced capital stock available for production in the next period satisfies

$$
k_{+}: \quad \frac{A}{w(\mathbf{s})}=\beta \int V_{k_{+}}\left(m_{+}, b_{+}, k_{+}, 0, \hat{\mathbf{s}}_{+}\right) \lambda\left(\mathbf{s}, d \hat{\mathbf{s}}_{+}\right)
$$

\subsubsection{Envelope conditions in the CM}

At an optimum, the envelope conditions for the agent's CM decision problem are as follows. The marginal value of money holdings upon entering the CM is

$$
W_{m}(m, b, k, l, \mathbf{s})=\frac{A \phi(\mathbf{s})}{w(\mathbf{s})},
$$

the marginal value of holding bonds upon entering the CM, respectively, are

$$
W_{b}(m, b, k, l, \mathbf{s})=\frac{A \phi(\mathbf{s})}{w(\mathbf{s})},
$$

and the marginal value of holding the each of the four types of capital stocks at the beginning of the CM are as follows. With respect to a Home agent's holding of capital stock in the Home country, the marginal $\mathrm{CM}$ value is

$$
W_{k}(m, b, k, l, \mathbf{s})=\frac{A}{w(\mathbf{s})}[1+r(\mathbf{s})]
$$

With respect to a Home agent's holding of credit in the Home country, the marginal CM value is

$$
W_{l}(m, b, k, l, \mathbf{s})=-\frac{A \phi(\mathbf{s})}{w(\mathbf{s})} .
$$

The envelope conditions (10)-(13) imply that, $W$ is linear in $(m, b, k, l)$, for each fixed aggregate state $\mathbf{s}$. So we can write $W$ as

$$
W(m, b, k, l, \mathbf{s})=W(0,0,0,0, \mathbf{s})+\frac{A}{w}[\phi(m+b)+(1+r) k] .
$$

\subsubsection{Firms}

Let $P_{h}$ be the Home currency price of the Home produced intermediate good, and $P_{y}$ be that of the Foreign produced intermediate good use by the Home final-good firm. The Home final-good firm solves

$$
\max _{y_{h}, y_{f}}\left\{\frac{G\left[y_{h}(\mathbf{s}), y_{f}(\mathbf{s})\right]}{\phi(\mathbf{s})}-P_{h}(\mathbf{s}) y_{h}(\mathbf{s})-P_{f}(\mathbf{s}) y_{f}(\mathbf{s})\right\} .
$$

The profit-maximizing conditions are:

$$
\phi(\mathbf{s}) P_{h}(\mathbf{s})=G_{y_{h}}\left[y_{h}(\mathbf{s}), y_{f}(\mathbf{s})\right]
$$


and

$$
\phi(\mathbf{s}) P_{f}(\mathbf{s})=G_{y_{f}}\left[y_{h}(\mathbf{s}), y_{f}(\mathbf{s})\right] .
$$

The Home intermediate goods producer solves

$$
\max _{H, K}\left\{P_{y_{h}}(\mathbf{s}) \cdot z F_{k}\left[K\left(\mathbf{s}_{-}\right), H(\mathbf{s})\right]-\frac{\left[\tilde{w}(\mathbf{s}) H(\mathbf{s})+\tilde{r}(\mathbf{s}) K\left(\mathbf{s}_{-}\right)\right]}{\phi(\mathbf{s})}\right\} .
$$

where the market for inputs to $F$ is perfectly competitive. Profit maximization is characterized by the usual first order conditions where capital and labor are paid a respective rental rate which equals their marginal products in every aggregate state s:

$$
\tilde{r}(\mathbf{s})=\phi(\mathbf{s}) P_{h}(\mathbf{s}) \cdot z F_{k}\left[K\left(\mathbf{s}_{-}\right), H(\mathbf{s})\right]
$$

and

$$
\tilde{w}(\mathbf{s})=\phi(\mathbf{s}) P_{h}(\mathbf{s}) \cdot z F_{H}\left[K\left(\mathbf{s}_{-}\right), H(\mathbf{s})\right],
$$

where

$$
H(\mathbf{s})=\int_{\mathbf{a}} H(\mathbf{a}, \mathbf{s}) \mu_{h}(\mathbf{s}, d \mathbf{a})
$$

is aggregate labor supply in the Home CM.

A foreign country's CM agent named $\left(m^{*}, b^{*}, k^{*}, l^{*}, \mathbf{s}\right)$ and its firm have a symmetric problem to (1)-(3), (15)-(16), and (17)-(18).

\subsection{Decentralized markets}

At the beginning of each $t \in \mathbb{N}$, an agent with state $(m, b, k, 0, \hat{\mathbf{s}})$ enters the DM. ${ }^{9}$ With a fixed probability $\sigma$ this agent is the buyer of the special good that some other agent produces, $q^{b}$, where the other agent (seller) is indexed by the state $(\tilde{\mathbf{a}}, \hat{\mathbf{s}}):=(\tilde{m}, \tilde{b}, \tilde{k}, 0, \hat{\mathbf{s}})$, but not vice-versa. With probability $\sigma \kappa$, the buyer parts with $d^{b}$ "dollars" and realizes a payoff of $u\left(q^{b}\right) \in \mathbb{R}$. The buyer then enters the day CM with a value of $W\left(m-d^{b}, b, k, 0, \mathbf{s}\right)$. With probability $\sigma(1-\kappa)$, the buyer does not use money, but takes out a nominal loan $l$, from the seller he meets, and realizes a payoff of $u\left(\breve{q}^{b}\right) \in \mathbb{R}$. The buyer then enters the day CM with a value of $W(m, b, k, l, \mathbf{s})$.

Symmetrically, with probability $\sigma \kappa$, agent $(m, b, k, 0, \hat{\mathbf{s}})$ can produce a special good $q^{s}$ which other buyers want to buy, but not vice-versa. This agent receives $d^{s}$ dollars in exchange for exerting a utility cost of production $c\left(q^{s} / z, k\right) \in \mathbb{R}_{+}$. Notice that capital obtained from the previous period's CM, $k$, accrues a return in the DM in the form of the marginal benefit to producing $q\left(q^{s}\right.$ or $\left.\breve{q}^{s}\right)$, i.e. $c_{k}(q / z, k) .{ }^{10}$ This seller then enters the day CM with a value of

\footnotetext{
${ }^{9}$ Note that $m$ implicitly includes any aggregate monetary transfer or injection from the government, which we denote later as $\iota(\hat{\mathbf{s}})$, so then, $m(\hat{\mathbf{s}})=m\left(\mathbf{s}_{-}\right)+\iota(\hat{\mathbf{s}})$.

${ }^{10}$ This feature was first introduced by Aruoba et al. (2011, Appendix A.1). The authors showed that whether
} 
$W\left(m+d^{s}, b, k, 0, \mathbf{s}\right)$. With probability $\sigma(1-\kappa)$, a seller may sell $\breve{q}^{s}$ by extending a loan $l$ to a matched buyer.

These four events described above are known as single-coincidence-of-wants meetings, where money is a portable medium of exchange in events that occur with probability $2 \sigma \kappa$, and where credit $l$ is the medium of exchange in events with probability $2 \sigma(1-\kappa)$. With probability $1-2 \sigma$, agent $(m, b, k, 0, \hat{\mathbf{s}})$ leaves the DM and enters the day with his assets intact, and begins his activity in the CM with value $W(m, b, k, 0, \mathbf{s})$. For simplicity, we assume the probability of a "double-coincidence" meeting, and hence the occurrence of pure barter, is zero.

Formally, an agent named $(m, b, k, 0, \hat{\mathbf{s}})$ has a value $V(m, b, k, 0, \hat{\mathbf{s}})$ at the beginning of the DM that satisfies the following problem:

$$
\begin{aligned}
V(m, b, k, 0, \hat{\mathbf{s}})=\sigma & V^{b}(m, b, k, 0, \hat{\mathbf{s}}) \\
& +\sigma V^{s}(m, b, k, 0, \hat{\mathbf{s}})+(1-2 \sigma) W(m, b, k, \mathbf{s}) .
\end{aligned}
$$

where, in general:

$$
\begin{aligned}
V^{b}(m, b, k, 0, \hat{\mathbf{s}})= & \kappa \int\left[u\left(q^{b}\right)+W\left(m-d^{b}, b, k, 0, \mathbf{s}\right)\right] \nu_{h}(d \tilde{\mathbf{a}}, \hat{\mathbf{s}}) \\
& +(1-\kappa) \int\left[u\left(\breve{q}^{b}\right)+W\left(m, b, k, l^{b}, \mathbf{s}\right)\right] \nu_{h}(d \tilde{\mathbf{a}}, \hat{\mathbf{s}}),
\end{aligned}
$$

and,

$$
\begin{aligned}
V^{s}(m, b, k, 0, \hat{\mathbf{s}})= & \kappa \int\left[-c\left(q^{s}, k\right)+W\left(m+d^{s}, b, k, 0, \mathbf{s}\right)\right] \nu_{h}(d \tilde{\mathbf{a}}, \hat{\mathbf{s}}) \\
& +(1-\kappa) \int\left[-c\left(\breve{q}^{s}, k\right)+W\left(m, b, k,-l^{s}, \mathbf{s}\right)\right] \nu_{h}(d \tilde{\mathbf{a}}, \hat{\mathbf{s}})
\end{aligned}
$$

are the value functions of ex-post buyer and sellers respectively.

\subsubsection{Walrasian price taking}

Consider a version of the DM where $\left(q^{b}, q^{s}, \tilde{p}, \breve{p}, \breve{q}^{b}, \breve{q}^{s}, l^{b}, l^{s}\right)$ are determined by Walrasian price taking. Then, we have

$$
\begin{aligned}
V^{b}(m, b, k, 0, \hat{\mathbf{s}}) & =\kappa \max _{q^{b} \in[0, m / \tilde{p}]}\left[u\left(q^{b}\right)+W\left(m-\tilde{p} q^{b}, b, k, 0, \mathbf{s}\right)\right] \\
& +(1-\kappa) \max _{\widetilde{q} \in\left[0, l^{b} / \tilde{p}\right]}\left[u\left(\breve{q}^{b}\right)+W\left(m, b, k, l^{b}, \mathbf{s}\right)\right],
\end{aligned}
$$

there exist two kinds of capital goods, for use in the DM and in the CM production, respectively, is of negligible quantitative consequence in their model. 
where $d^{b}=\tilde{p} q^{b}$, and,

$$
\begin{aligned}
V^{s}(m, b, k, 0, \hat{\mathbf{s}}) & =\kappa \max _{q^{s}}\left[-c\left(q^{s} / z, k\right)+W\left(m+\tilde{p} q^{s}, b, k, 0, \mathbf{s}\right)\right] \\
& +(1-\kappa) \max _{\tilde{q}^{s}}\left[-c\left(\breve{q}^{s} / z, k\right)+W\left(m, b, k,-l^{s}, \mathbf{s}\right)\right]
\end{aligned}
$$

where $d^{s}=\tilde{p} q^{s}, \tilde{p}$ and $\breve{p}$ are the respective prices of a special good in anonymous and monitored trades, taken as given by all buyers and sellers.

\subsection{Government}

New money is injected at the end of the period in the CM. ${ }^{11}$ Specifically, the monetary authority follows a monetary supply rule:

$$
M(\mathbf{s})=\exp (\psi) M\left(\mathbf{s}_{-}\right),
$$

where $\exp \{\psi\}-1$ is the one-period money supply growth rate between time $t$ and $t+1$. Assume that $\left(\exp \left(\psi_{t}\right)\right)_{t \in \mathbb{N}}$ follows a Markov process that lives in the compact set $[1, N]$, with $N<+\infty$. We define this process later.

Government expenditure $G^{d}$ is financed by lump-sum taxes/transfers, seigniorage and consumption, labor and capital tax revenue:

$$
G^{d}(\mathbf{s})=\left[T(\mathbf{s})+\left(M(\mathbf{s})-M\left(\mathbf{s}_{-}\right)\right) \phi(\mathbf{s})\right]+\tau_{X} X(\mathbf{s})+\tau_{H} H(\mathbf{s})+\tau_{K}(\tilde{r}(\mathbf{s})-\delta) K\left(\mathbf{s}_{-}\right) .
$$

We assume that $T(\mathbf{s})=-\left(M(\mathbf{s})-M\left(\mathbf{s}_{-}\right)\right) \phi(\mathbf{s})$.

\section{Stationary Markov Monetary Equilibrium}

In this section, we state a key result which is just an extension of Lagos and Wright (2005) to environments with aggregate uncertainty. ${ }^{12}$ In an equilibrium, the endogenous distribution of agents' asset holdings is degenerate at the start of each period (and hence DM), such that all agents in each country choose the same allocations that depend only on the global state. We further characterize the equilibrium conditions in the DM and list the conditions for market clearing in the CM. We then define the elements that constitute a stationary Markov monetary equilibrium.

In general, because of the random meeting technology in the DM, we will need to track the history of aggregate distribution of assets held by agents in any equilibrium where money

\footnotetext{
${ }^{11}$ This is merely for mathematical convenience, so that within each DM, agents do not have to deal with a stochastic total payoff function, $W$.

${ }^{12} \mathrm{~A}$ proof is available upon request from the authors.
} 
has value. However, because of the quasi-linear assumption on each agent's per-period payoff function, it can be shown that in equilibrium asset holdings at the beginning of each $t \in \mathbb{N}$ are identical across all agents within each country $i$, so that,

$$
\begin{aligned}
(m, b, k, 0)(\mathbf{s}) & =\int(m, b, k, 0) \nu_{i}(\hat{\mathbf{s}}, d m, d b, d k, d l) \\
& :=:(M, B, K, 0)(\hat{\mathbf{s}}) \\
& =:(M, B, K, 0)(\mathbf{z}) .
\end{aligned}
$$

for each $i \in\{h, f\}$, for all $\hat{\mathbf{s}}$. This implies that we can explicitly write $\nu(\hat{\mathbf{s}}, \cdot)$ as $\nu(\mathbf{z}, \cdot)$, and furthermore, for every $\mathbf{z}$, and every $A \in \mathcal{B}_{i}(\mathbf{z})$,

$$
\nu_{i}(\mathbf{z}, A)=\left\{\begin{array}{ll}
1 & \text { if }(m, b, k, 0)=(M, B, K, 0) \in A \\
0 & \text { otherwise }
\end{array} .\right.
$$

However, we can see that even if $\nu_{i}(\mathbf{z}, \cdot)$ is degenerate at the end of the $\mathrm{CM}, \mu_{i}(\mathbf{z}, \cdot)$ is not. Thus, explicitly, agents at the beginning of each $\mathrm{CM}$ will still face an aggregate state variable $\mathbf{s}$ that contains a non-degenerate distribution of individual states. Specifically, the non-degeneracy is along the dimension of money holdings out of the DM.

\subsection{DM competitive pricing and equilibrium decisions}

In equilibrium, the constraints $d \leq m$, and $l \leq \breve{p} \breve{q}$ bind, and $q^{b}=q^{s}=q$. Thus for the $\sigma \kappa$ proportion of agents who are sellers that meet buyers and trade with money, we have the equilibrium condition that the marginal utility value to the buyer of a unit of the home currency (for buying $q$ ), is equal to the marginal utility cost of production of the DM seller:

$$
\frac{A \phi}{w} M=\frac{1}{z} c_{q}(q / z, K) q \equiv g(q, K, z) .
$$

Note that $\tilde{p}=M / q$ in equilibrium. If we assume alternative DM protocols for determining the terms of trade - e.g. generalized Nash bargaining - then the function $g$, which would represent a bilateral buyer-seller sharing function, will be quite different. ${ }^{13}$

For the $\sigma(1-\kappa)$ proportion of buyers and sellers, we have:

$$
\frac{A \phi}{w} l=\frac{1}{z} c_{q}(\breve{q} / z, K) \breve{q} \equiv g(\breve{q}, K, z) .
$$

Since by assumption contracts are enforceable for these agents, then credit attains the first

\footnotetext{
${ }^{13}$ These alternatives are considered quantitatively later, and discussed in detail in a separate Appendix available upon request.
} 
best DM allocation in terms of $\breve{q}$ satisfying

$$
u_{q}(\breve{q})=\frac{1}{z} c_{q}(\breve{q} / z, K)
$$

Therefore we can substitute out credit in the equilibrium conditions later, using

$$
l=\frac{w u_{q}(\breve{q}) \breve{q}}{A \phi}
$$

\subsection{Envelope conditions in the DM}

At an interior optimum consistent with equilibrium, we have the following envelope conditions. Utilizing the linearity of $W$, the marginal value of money at the beginning of the DM is

$$
V_{M}(M, B, K, 0, \hat{\mathbf{s}})=\frac{A \phi}{w}\left[(1-\sigma \kappa)+\sigma \kappa \frac{z \cdot u_{q}(q)}{c_{q}(q / z, K)}\right]>0 .
$$

The marginal value of the state-contingent money claims at the beginning of the DM is

$$
V_{B}(M, B, K, 0, \hat{\mathbf{s}})=W_{b}(M, B, K, 0, \mathbf{s})=\frac{A \phi}{w} .
$$

The DM marginal value of the capital stock, is

$$
V_{K}(M, B, K, 0, \hat{\mathbf{s}})=\frac{A \phi}{w}(1+r)-\sigma \kappa \gamma(q, K, z)-\sigma(1-\kappa) \gamma(\breve{q}, K, z)>0,
$$

where

$$
\gamma(q, K, z)=c_{K}(q / z, K)<0 .
$$

The function $\gamma$ is strictly negative due to two effects that capture the reduction in marginal cost of production in the DM. The first term on the right of (30) is the indirect effect on marginal cost through the effect of an additional capital stock on the terms of trade $q$.

\subsection{Market clearing in the $\mathrm{CM}$}

In an equilibrium, since agents within each country choose the same asset holdings, i.e. $(m, b, k)=(M, B, K)$, then they do not borrow from, or, lend to each other, only countries lend to each other. Therefore, in the global equilibrium, state-contingent money claims by Home and Foreign have zero excess demand:

$$
B(\mathbf{s})+B^{*}(\mathbf{s})=0
$$

in every state $\mathbf{s}$. 
The Home resource constraint is given by

$$
G\left[y_{h}(\mathbf{s}), y_{f}(\mathbf{s})\right]=X(\mathbf{s})+I(\mathbf{s})+G^{d}(\mathbf{s}),
$$

where $I(\mathbf{s})=K(\mathbf{s})-(1-\delta) K\left(\mathbf{s}_{-}\right)$is domestic capital investment.

The Foreign resource constraint is given by

$$
G\left[y_{f}^{*}(\mathbf{s}), y_{h}^{*}(\mathbf{s})\right]=X^{*}(\mathbf{s})+I^{*}(\mathbf{s})+G^{d *}(\mathbf{s})
$$

where $I^{*}(\mathbf{s})=K^{*}(\mathbf{s})-(1-\delta) K^{*}\left(\mathbf{s}_{-}\right)$is the Foreign country's investment in its own capital stock, and, government spending $G^{d *}$ is given by

$$
\begin{aligned}
G^{d *}(\mathbf{s})=\left[T^{*}(\mathbf{s})\right. & \left.+\left(M^{*}(\mathbf{s})-M^{*}\left(\mathbf{s}_{-}\right)\right) \phi^{*}(\mathbf{s})\right] \\
& +\tau_{X} X^{*}(\mathbf{s})+\tau_{H} H^{*}(\mathbf{s})+\tau_{K}\left(\tilde{r}^{*}(\mathbf{s})-\delta\right) K^{*}\left(\mathbf{s}_{-}\right) .
\end{aligned}
$$

We also assume that $T^{*}(\mathbf{s})=-\left(M^{*}(\mathbf{s})-M^{*}\left(\mathbf{s}_{-}\right)\right) \phi(\mathbf{s})$.

Market clearing for the intermediate goods must hold:

$$
\begin{aligned}
z F\left[K\left(\mathbf{s}_{-}\right), H(\mathbf{s})\right] & =y_{h}(\mathbf{s})+y_{h}^{*}(\mathbf{s}) \\
z^{*} F\left[K^{*}\left(\mathbf{s}_{-}\right), H^{*}(\mathbf{s})\right] & =y_{f}^{*}(\mathbf{s})+y_{f}(\mathbf{s})
\end{aligned}
$$

Definition 1 A stationary Markov monetary equilibrium (SME), given any feasible monetary policy rule $\left(\psi, \psi^{*}\right)$, is a set of time-invariant maps consisting of

E1. strictly positive pricing functions $\left(\phi, \phi^{*}, e\right)$ and $\left(w, r, w^{*}, r^{*}, Q\right)$,

E2. transition laws $(\mathcal{G}, \varphi)$ and $\left(\mathcal{G}^{*}, \varphi^{*}\right)$,

E3. value functions $V, W$ and $V^{*}, W^{*}$,

E4. CM decision rules $\left(X, X^{*}, m, m^{*}, b, k, b^{*}, k^{*}\right)$, and

E5. DM terms of trade (decision rules), $(d, q, \breve{q})$ and $\left(d^{*}, q^{*}, \breve{q}^{*}\right)$, such that:

1. given prices (E1), the value functions $V$ and $W$ satisfy the functional equations (1), (2), (3), and (19) and symmetrically $V^{*}, W^{*}$ solve the Foreign country counterpart problems;

2. given the value functions $V$ and $W$, and prices (E1), the decision rules E4 solve (1), (2), (3) in the $C M$, for the Home country and symmetrically for the Foreign country, given $V^{*}$ and $W^{*}$; 
3. Firms optimize: (17) and (18);

4. given the value functions $W$ and $V$, the decision rules E5 solve and (23), (25), and (26) in the DM, and symmetrically for the Foreign country, given $W^{*}$;

5. The government budget constraint (21) is satisfied for Home and symmetrically for Foreign.

6. Markets clear in the $C M$ and $C M^{*}$ : (31), (32) and (33), where $m=M, b=B$ and $k=K$, and $m^{*}=M^{*}, b^{*}=B^{*}$ and $k^{*}=K^{*}$.

\subsection{Other variable definitions}

Since the model features a DM sector that is akin to a nontraded goods sector, we will define a relevant price index, which will be used toward the construction of a real exchange rate definition. First we define a DM price index as the convex combination of the pricing outcome in monetary and credit trades:

$$
p_{D M}:=\kappa \tilde{p}+(1-\kappa) \breve{p} .
$$

The foreign counterpart will be $p_{D M}^{*}$. Denote the aggregate DM consumption as

$$
q_{D M}:=\kappa q+(1-\kappa) \breve{q} .
$$

Now we can define our measure of aggregate price index (or output deflator) as

$$
P_{Y}=\zeta \phi^{-1}+(1-\zeta) p_{D M},
$$

where

$$
\zeta=\frac{X}{X+\sigma q_{D M}},
$$

is the $\mathrm{CM}$ consumption share in total domestic consumption. Note that this share is timevarying in the sense that it is dependent on the aggregate state $\mathbf{s}$. The foreign price index is defined analogously as $P_{Y}^{*}$. Now we define the real exchange rate as

$$
R E R(\mathbf{s}):=\frac{e(\mathbf{s}) P_{Y}^{*}(\mathbf{s})}{P_{Y}(\mathbf{s})} .
$$

\section{Implications for Exchange Rate Dynamics}

We now analyze the implication of the assumption of anonymity $(0<\kappa \leq 1)$, for exchange rate dynamics. For ease of notation and exposition, and without loss of generality, we consider $\kappa=1$ (i.e. extreme anonymity in the DM) for now and $\tau_{X}=\tau_{H}=\tau_{K}=0$. Using the 
first-order conditions in the CM and DM, the corresponding envelope conditions, and imposing equilibrium, we can derive a set of stochastic Euler functional equations necessary for characterizing a stationary Markov monetary equilibrium (SME). We can write the SME conditions as ones that characterize the solutions as s-dependent processes. ${ }^{14}$

First, from (6), we can easily deduce that in equilibrium, $X(\mathbf{a}, \mathbf{s})=X(\mathbf{s})$, and, $X^{*}\left(\mathbf{a}^{*}, \mathbf{s}\right)=$ $X^{*}(\mathbf{s})$, for all s. Also, $q(m, k, \mathbf{s})=q(M, K, \mathbf{s}) \equiv q(\mathbf{s})$, and, $q^{*}\left(m^{*}, k^{*}, \mathbf{s}\right)=q^{*}\left(M^{*}, K^{*}, \mathbf{s}\right) \equiv$ $q^{*}(\mathbf{s})$. Together with (7) and (27), we have the SME version of the Euler functional equation for optimal money holdings in the Home country:

$$
U_{X}[X(\mathbf{s})]=\beta \mathbb{E}_{\lambda}\left\{U_{X}\left[X\left(\mathbf{s}_{+}\right)\right] \frac{\phi\left(\mathbf{s}_{+}\right)}{\phi(\mathbf{s})}\left[(1-\sigma)+\sigma \frac{z_{+} u_{q}\left[q\left(\mathbf{s}_{+}\right)\right]}{c_{q}\left[q\left(\mathbf{s}_{+}\right) / z_{+}, K(\mathbf{s})\right]}\right]\right\},
$$

where, $\mathbb{E}_{\lambda}$ denotes the expectation operator with respect to the conditional distribution $\lambda(\mathbf{s}, \cdot)$, and, the term in the square brackets is the expected (with respect to $\nu_{h}$ ) one-period nominal gross return on money holding. There is an equivalent condition for the foreign country.

Second, since in equilibrium, $X(\mathbf{a}, \mathbf{s})=X(\mathbf{s})$ for all $\mathbf{s}$, along with (8) and (28), we then have an Euler equation for optimal Home bond holdings:

$$
\begin{aligned}
Q\left(\mathbf{s}_{+} \mid \mathbf{s}\right):= & {\left[\int_{\mathbf{a}_{+}} Q\left(\mathbf{a}_{+}, \mathbf{s}_{+} \mid \mathbf{a}, \mathbf{s}\right) \mu_{h}\left(\mathbf{s}_{+}, d \mathbf{a}_{+}\right)\right] \lambda\left(\mathbf{s}, d \mathbf{s}_{+}\right) } \\
& =\beta \frac{U_{X}\left[X\left(\mathbf{s}_{+}\right)\right]}{U_{X}[X(\mathbf{s})]} \frac{\phi\left(\mathbf{s}_{+}\right)}{\phi(\mathbf{s})} \lambda\left(\mathbf{s}, d \mathbf{s}_{+}\right), \quad \forall \mathbf{s}, \mathbf{s}_{+} .
\end{aligned}
$$

Third, Foreign agents would also have a first order condition for bonds similar to (38), which, in Home currency terms is:

$$
\begin{aligned}
Q\left(\mathbf{s}_{+} \mid \mathbf{s}\right):= & {\left[\int_{\mathbf{a}_{+}^{*}} Q\left(\mathbf{a}_{+}^{*}, \mathbf{s}_{+} \mid \mathbf{a}, \mathbf{s}\right) \mu_{f}\left(\mathbf{s}_{+}, d \mathbf{a}_{+}^{*}\right)\right] \lambda\left(\mathbf{s}, d \mathbf{s}_{+}\right) } \\
& =\beta \frac{U_{X}\left[X^{*}\left(\mathbf{s}_{+}\right)\right]}{U_{X}\left[X^{*}(\mathbf{s})\right]} \frac{\phi^{*}\left(\mathbf{s}_{+}\right)}{\phi^{*}(\mathbf{s})} \frac{e(\mathbf{s})}{e\left(\mathbf{s}_{+}\right)} \lambda\left(\mathbf{s}, d \mathbf{s}_{+}\right), \quad \forall \mathbf{s}, \mathbf{s}_{+} .
\end{aligned}
$$

From (6), (9) and knowing $V_{K}$, we have an Euler equation for optimal Home capital holdings:

$$
\begin{aligned}
& U_{X}[X(\mathbf{s})]= \\
& \quad \beta \mathbb{E}_{\lambda}\left\{U_{X}\left[X\left(\mathbf{s}_{+}\right)\right]\left[\left(1+r\left(\mathbf{s}_{+}\right)-\delta\right)-\sigma \frac{\gamma\left[q\left(\mathbf{s}_{+}\right), K(\mathbf{s}), z_{+}\right]}{U_{X}\left[X\left(\mathbf{s}_{+}\right)\right]}\right]\right\} .
\end{aligned}
$$

\footnotetext{
${ }^{14}$ The full details are given in a separate Appendix available from the authors. Recall that in any equilibrium, agents end up choosing the same asset allocations regardless of their personal state. Thus, with a slight abuse of notation, we drop the dependency on aggregate state variables such as $\mu_{i}(\mathbf{s}, \cdot), i \in\{h, f\}$, from the definition of $\mathbf{s}$ in equilibrium. In other words, the Euler equations below will have the appearance as though they were-and indeed they are - characterizing equilibrium of some representative agent model.
} 
There is also a symmetric characterization for the foreign country.

\subsection{Inspecting the mechanism}

Equating (38) and (39) and iterating, we have

$$
\frac{U_{X}[X(\mathbf{s})]}{U_{X}\left[X\left(\mathbf{s}_{0}\right)\right]} \frac{\phi(\mathbf{s})}{\phi\left(\mathbf{s}_{0}\right)}=\frac{U_{X}\left[X^{*}(\mathbf{s})\right]}{U_{X}\left[X^{*}\left(\mathbf{s}_{0}\right)\right]} \frac{e\left(\mathbf{s}_{0}\right)}{e(\mathbf{s})} \frac{\phi^{*}(\mathbf{s})}{\phi^{*}\left(\mathbf{s}_{0}\right)},
$$

where $\mathbf{s}_{0}$ is the initial aggregate state. Assume that the initial condition, given by

$$
\kappa_{0}:=\frac{e\left(\mathbf{s}_{0}\right) U_{X}\left[X\left(\mathbf{s}_{0}\right)\right] \phi\left(\mathbf{s}_{0}\right)}{U_{X}\left[X^{*}\left(\mathbf{s}_{0}\right)\right] \phi^{*}\left(\mathbf{s}_{0}\right)}
$$

is fixed.

We can re-write the expression in (41) as the equilibrium determination of the nominal exchange rate:

$$
e(\mathbf{s})=\kappa_{0} \frac{U_{X}\left[X^{*}(\mathbf{s})\right]}{U_{X}[X(\mathbf{s})]} \frac{\phi^{*}(\mathbf{s})}{\phi(\mathbf{s})} .
$$

This warrants some remark. Up to this point, in terms of equilibrium complete state-contingent money claims, we have derived a standard complete markets (in terms of the $\mathrm{CM}$ ) result for the nominal exchange rate (see e.g. Chari et al., 2002). What equation (42) says is that the nominal exchange rate, at each state of the world, is proportional to the within-period relative value of the marginal rate of substitution of the general good between Home and Foreign consumers.

Note however, in equilibrium, the DM price-taking protocol implies that buyers' marginal utility value of holding domestic currency must equal sellers' marginal utility cost of producing good $q$, where by anonymity, must be purchased with money:

$$
U_{X}[X(\mathbf{s})] \phi(\mathbf{s}) M(\mathbf{s})=\frac{1}{z} c_{q}\left(\frac{q(\mathbf{s})}{z}, K\left(\mathbf{s}_{-}\right)\right) q(\mathbf{s}) \equiv g\left[q(\mathbf{s}), K\left(\mathbf{s}_{-}\right), z\right] .
$$

In terms of stationary variables - i.e. normalizing by $M\left(\mathbf{s}_{-}\right)$- and assuming logarithmic utility for $U$, we have:

$$
\frac{\hat{\phi}(\mathbf{s})}{X(\mathbf{s})}=\frac{1}{\exp \left\{\psi_{t}\right\}} c_{q}\left(\frac{q(\mathbf{s})}{z}, K\left(\mathbf{s}_{-}\right)\right) \frac{q(\mathbf{s})}{z} \equiv \frac{1}{\exp \left\{\psi_{t}\right\}} g\left[q(\mathbf{s}), K\left(\mathbf{s}_{-}\right), z\right],
$$

where $\hat{\phi}(\mathbf{s}):=\phi(\mathbf{s}) M\left(\mathbf{s}_{-}\right)$and $M(\mathbf{s}) / M\left(\mathbf{s}_{-}\right)=\exp \left\{\psi_{t}\right\}$.

In contrast now, consider a version of our model where money is introduced via a cash-inadvanced (CIA) constraint. In a monetary equilibrium where the CIA constraint binds almost surely, we would have:

$$
\frac{\hat{\phi}(\mathbf{s})}{X(\mathbf{s})}=\frac{1}{\exp \left\{\psi_{t}\right\}} .
$$


The interpretation in the CIA version is obviously quite different. In such an economy, agents are constrained to hold money to buy goods by assumption. Equation (45) implies that a positive increase in money supply (on the right) must be followed by a virtually one-for-one increase in the price level (or decrease in the value of a dollar, $\hat{\phi}$ ), if equilibrium consumption $X$ is smooth (or equivalently if agents are risk-averse and markets are complete). In short, the relative price of a unit of $X$ is extremely flexible in response to a monetary shock. If so, from the nominal exchange rate determination condition in (42), we can immediately deduce that there would be very little volatility in the nominal exchange rate. Hence there would be very little connection between the nominal and the real exchange rates as well, by the definition of the real exchange rate. ${ }^{15}$

Consider now our model with extreme anonymity $(\kappa=1)$. Anonymity implies that the equilibrium condition (44) must hold. With log utility, we can study part of the model's mechanism by contrasting between the equilibrium condition (44) and a hypothetical CIA constraint (45). In contrast, even in the presence of consumption smoothing, the DM equilibrium pricing condition (44) implies that an increase in money supply need not be followed by a one-forone increase in the price level, or a decrease in the value of money. Holding the conditional expectations on the right of (37) constant, a positive monetary injection means that current $q$ will increase, on the left side of the equilibrium money Euler equation (37). As current $q$ increases immediately, this has an opposing effect to an increase in money supply. That is, on the one hand, an increase in money supply has a tendency to reduce the marginal utility value of holding a dollar (the left side of (44)), an increase in $q$ tends to increase the utility value of that dollar purchasing the special good $q$ (the right side of (44)). Depending on the nature of the DM pricing protocol and parametrization - i.e. the shape of $g$, it may be that the value of a dollar $\hat{\phi}$ need not fall as much as the increase in money supply. In other words, it may be possible that the equilibrium pricing process will appear rather rigid or unresponsive as an equilibrium outcome, rather than being an assumption.

Consider also a supply-side or technology shock, $z$. An increase in $z$, has a tendency to raise the current marginal product of labor and hence labor demand in the CM. Equating (6) and (18), we have a condition for equilibrium labor market clearing in the CM. From this, we can see that if consumption increases but by not as much as income, then labor allocation would also increase. This would imply an increase in current CM investment into productive capital stock next period. Since $c(q / z, K)$ is the dual cost function to an homogeneous of degree one production technology in the DM, we can deduce that an increase in $z$ will lower the marginal cost of producing $q$. This will, in turn, lower the term on the right of the equilibrium monetary pricing condition (44). However, the technology shock also affects the left side of (44) via raising the marginal product of labor, and hence lowering the marginal utility of $X$,

\footnotetext{
${ }^{15}$ This point has previously been verified by the earlier work of Schlagenhauf and Wrase (1995) in the context of a two-country CIA monetary model.
} 
$U_{X}(X)$. Again, depending on the shape of $g$, the value of a dollar, $\hat{\phi}$, need not be so responsive to a technology shock. (This is further corroborated in our numerical results in Section 6 below.) Therefore, consistent with the nominal exchange rate determination condition (42), the nominal exchange rate ought to be quite volatile too. Since the real exchange rate in our two-sector model is defined by (36), we would expect the real exchange rate to co-move with the nominal exchange rate.

In the next sections, we will validate these equilibrium implications for the exchange rate dynamics.

\section{Computational Exercise}

For our numerical exercise, we consider the following specific functions to represent the model primitives. In the CM, per-period preferences and technology are represented by

$$
U(X)=B \frac{X^{1-\gamma}-1}{1-\gamma}, \quad z F(K, H)=z K^{\alpha} H^{1-\alpha},
$$

respectively, where $B>0, \gamma>0$, and $\alpha \in(0,1)$. The symmetric description holds for the Foreign country. Note however, the notation for the final goods production function $G$ is such that

$$
G\left(y_{h}, y_{f}\right)=\left[\vartheta\left(y_{h}\right)^{\frac{1}{\epsilon}}+(1-\vartheta)\left(y_{f}\right)^{\frac{1}{\epsilon}}\right]^{\epsilon}
$$

for the Home country, and,

$$
G\left(y_{f}^{*}, y_{h}^{*}\right)=\left[\vartheta\left(y_{f}^{*}\right)^{\frac{1}{\epsilon}}+(1-\vartheta)\left(y_{h}^{*}\right)^{\frac{1}{\epsilon}}\right]^{\epsilon}
$$

for the Foreign country, where $\vartheta \in(0,1)$ and $-\infty \leq 1 / \epsilon \leq 1$. The elasticity of substitution between the inputs to $G$ is given by $\sigma_{\epsilon}=\epsilon /(\epsilon-1)$. These functional forms are quite standard in models with international trade in intermediate goods (see e.g. Heathcote and Perri, 2002; Chari et al., 2002).

In the DM, per-period preferences and technology are respectively represented by

$$
u(q)=C \frac{(q+\underline{q})^{1-\eta}-b^{1-\eta}}{1-\eta}, \quad c(q, K)=q^{\varpi}(K)^{1-\varpi}
$$

where $C=1$, without loss of generality, $\eta>0$ and $\varpi \geq 1$. We set $\underline{q}=0$ if DM trade is determined by competitive price taking, and $\underline{q} \searrow 0$ in the case of DM bargaining. The latter assumption is required for a well-defined outside-option value in the bargaining problem (see e.g. Lagos and Wright, 2005). 
Table 1: Calibration and Parameterization

\begin{tabular}{lrl}
\hline \hline Parameter & Values & Remarks \\
\hline$\beta$ & 0.99 & Fixed \\
$\eta=\gamma$ & 1 & Fixed \\
$\delta$ & 0.025 & $I / K$ \\
$\alpha$ & $1 / 3$ & Total capital income share, $1 / 3$ \\
$A$ & 0.4858 & Total labor hours fraction, $1 / 3$ \\
$\varpi$ & 1.2766 & $K / Y=8.92$ per quarter $(2.23$ per annum) \\
$\sigma$ & 0.13 & Real money demand interest elasticity, $-0.23(\mathrm{AWW})$ \\
$B$ & 0.1686 & Non-traded good consumption share, 0.50 \\
$\vartheta$ & 0.9397 & Share of imports in net exports (CKM) \\
$\epsilon$ & 3 & Estimated, CKM, BKK \\
$\kappa$ & 0.85 & Estimated, AWW \\
$\tau_{K}$ & 0.548 & Estimated, AWW \\
$\tau_{H}$ & 0.242 & Estimated, AWW \\
$\tau_{X}$ & 0.069 & Estimated, AWW \\
\hline \hline Notes: & &
\end{tabular}

(a) Aruoba et al. (2011): (AWW)

(b) Backus et al. (1994): (BKK).

(c) Chari et al. (2002): (CKM).

\subsection{Baseline model calibration}

Table 1 summarizes the baseline parameter values for the model. To discipline our numerical exercise, we calibrate the model with a quarterly frequency to match long run stylized facts. First, we discuss parameters that can be easily estimated or fixed indepedently. Similar to Aruoba et al. (2011), we calibrate $\alpha$ to match the target of labor share in output, which is about 0.7 in the data (see also Aruoba, 2010). We fix $\delta=0.1$ as estimated in Heathcote and Perri (2002) for a two country model. Following Aruoba et al. (2011) and Aruoba (2010), we calibrate $\sigma$ to match the long-run money demand semi-elasticity with respect to the nominal interest rate, where money is defined by M1 for the U.S. This elasticity is about -0.23 . The risk aversion parameters $\eta$ and $\gamma$ imply that both $U$ and $u$ are natural $\log$ functions of $X$ and $q$, respectively. This restriction is required for the baseline model to have a balanced growth path, since the per-period utility function is linearly separable in consumption and leisure (see Waller, 2010). The constant marginal taxes on capital, labor and CM-consumption, $\left(\tau_{K}, \tau_{H}, \tau_{X}\right)=(0.548,0.242,0.069)$, are chosen as in Aruoba et al. (2011). The estimate of $\vartheta$ is from Backus et al. (1994).

Second, we calibrate simultaneously the remaining parameters $(A, B, \varpi)$ to match the targets of proportion of total hours worked (DM and CM aggregate), $H_{t o t}$, a measure of non-traded consumption goods share in total consumption, NTS, and the long run capital output ratio, $K / Y$. The value of $H_{t o t}$ is roughly 0.33 , which is standard. This value can be thought of as pinning down the marginal utility of labour parameter $A$. $B$ is calibrated, in this model, to match a DM consumption (interpreted as a nontradable good in this model) share of total consumption to be close to 0.50 for the U.S., a share estimated by Stockman and Tesar (1995). This is in contrast to the closed-economy models in Aruoba et al. (2011) and Aruoba (2010), where intuitively, $B$ is calibrated to match the velocity of money. The target capital-output 
Table 2: Percentage standard deviation relative to output

\begin{tabular}{lccc|cc}
\hline \hline & Data & PT & (\% data) & CKM (\% data) & HP (\% data) \\
\hline Nominal E.R., $e$ & 3.34 & 4.82 & 144 & {$[1.5,100]$} & n.a. \\
Real E.R., $R E R$ & 3.36 & 2.34 & 70 & {$[1.1,114]$} & 100 \\
Consumption, $C$ & 0.72 & 0.61 & 85 & {$[100,111]$} & {$[63,65]$} \\
Investment, $I$ & 2.70 & 1.82 & 67 & {$[54,84]$} & {$[73,98]$} \\
Hours, $H_{\text {tot }}$ & 0.83 & 0.46 & 55 & {$[224,233]$} & {$[42,48]$} \\
\hline \hline
\end{tabular}

(a) Percentage of authors' data statistics accounted for by authors' models.*

(b) Chari et al. (2002) (CKM).

(c) Heathcote and Perri (2002) (HP) model real business cycles.

ratio, $K / Y$, is 2.23 in annual terms. Given other parameters, this ratio can be thought of as pinning down the calibration for $\varpi$ from the Euler equation characterizing equilibrium capital accumulation along the steady state path. The calibrated value of $\varpi>1$, implies that the more capital is installed for use in the DM production, the lower the cost of producing a unit of DM output $q$. By duality, this implies that capital is a complementary input to labor effort in DM production.

In the baseline model, we assume that all the TFP levels (and their shocks), in both CM and DM, are uncorrelated with each other (see also Chari et al., 2002). In parameterizing the exogenous TFP autocorrelation parameters $\left(\rho_{Z}, \rho_{Z^{*}}\right)$ we follow Chari et al. (2002). The money supply growth stochastic processes are the estimates from Schlagenhauf and Wrase (1995).

\section{International business cycle features}

In this section, we discuss the business cycle dynamics of the calibrated baseline model. We report the quantitative predictions of our benchmark model (labelled "PT" in the tables) relative to a class of business cycle models with sticky prices considered by Chari et al. (2002) (labelled CKM in the tables), and a real business cycle model of Heathcote and Perri (2002) (HP in the tables).

Hereinafter, when we refer to aggregate or total consumption $(C)$, output $(Y)$ or labor $\left(H_{t o t}\right)$ variables, we mean the real allocations of these variables in both the DM and the CM in our model, where the implicit deflator is the output deflator $P_{Y}$, as constructed previously in Section 3.4. Aggregate investment $(I)$ and net exports $(N X)$ will be real variables in terms of aggregate goods with price index $P_{Y}$.

As we can see from Table 2, the benchmark model can account for the volatilities of the key business cycle data for the U.S. quite well. ${ }^{16}$ In particular, the model can account for up to $85 \%$ of aggregate consumption volatility, $67 \%$ of the volatility in domestic investment, and about $55 \%$ of total labor volatility. The model over-predicts the nominal exchange rate

\footnotetext{
${ }^{16}$ Appendix A contains the description of our data.
} 
Table 3: Autocorrelations and cross-correlations

\begin{tabular}{lccc|c}
\hline \hline & Data & PT & PT (\% data) & CKM (\% data) \\
\hline Autocorrelation: & & & & \\
Nominal E.R., $e$ & 0.83 & 0.66 & 80 & {$[53,97]$} \\
Real E.R., RER & 0.84 & 0.66 & 79 & {$[58,93]$} \\
Consumption, $C$ & 0.87 & 0.82 & 94 & {$[3.4,75]$} \\
Investment, I & 0.90 & 0.78 & 87 & {$[3.3,75]$} \\
Hours, Htot & 0.94 & 0.92 & 98 & {$[3.3,77]$} \\
Output, Y & 0.89 & 0.79 & 89 & {$[3.4,80]$} \\
Contemporaneous correlation: & & & & \\
(RER, $)$ & 0.99 & 0.99 & 100 & {$[71,99]$} \\
$(R E R, N X)$ & 0.14 & 0.17 & 121 & {$[-436,214]$} \\
\hline \hline
\end{tabular}

(a) Percentage of authors' data statistics accounted for by authors' models.*

(b) A negative sign indicates a counterfactual direction in the model-data accounting.*

(c) Chari et al. (2002) (CKM) consider several model variations.

(d) Heathcote and Perri (2002) (HP) did not report these statistics.

volatility by $44 \%$ but accounts for a substantial amount of the real exchange rate volatility (70\%). Consider the last two columns in Table 2. Relative to previous accounts by Chari et al. (2002) (various versions of sticky price and/or wages model and with/without mutiple shocks or Taylor rule) and Heathcote and Perri (2002) (real business cycle model with exogenous financial autarky), our more does quite well.

Overall, in terms of the nominal and real exchange rate volatilities, the model is able to reproduce qualitatively the observation that both exchange rates are much more volatile than U.S. GDP. As opposed to Chari et al. (2002) and Heathcote and Perri (2002), our benchmark model does not rely on large relative risk aversion parameters (viz. we assume log utility), sticky prices nor imperfections in international risk sharing to generate volatility. ${ }^{17}$ Furthermore, in contrast, standard flexible price two-country CIA models (see Schlagenhauf and Wrase, 1995) are unable to reproduce any realistic volatilities in the real and nominal exchange rates.

Next, consider the first order autocorrelation coefficients of the equilibrium processes in Table 3. In terms of consumption, investment, labor allocation, and output, the model matches the empirical persistence in the data quite well, and comparable to Chari et al. (2002). However, in terms of the real and nominal exchange rates, the model under-accounts for the persistence observed in the data by about $20 \%$. Nevertheless, the baseline model is able to do just as well as some of the models considered in Chari et al. (2002), without requiring any exogenous sticky-price assumption.

In terms of the other open-economy correlations in the data, the model is able to account for the mild positive correlation between the real exchange rate and net exports in the data. Moreover, the model is able to generate a real-nominal exchange rate correlation that is very

\footnotetext{
${ }^{17}$ On the other hand, the competitive equilibrium in our model features incomplete markets as a result of idiosyncratic shocks to agent types each period as they enter the DM. Since there is a link between the DM and $\mathrm{CM}$ outcomes via capital, not all consumption risk can be fully insured.
} 
close to the data. To see why, we consider the partial explanations given in Figures 2 and 3. Figure 2 depicts the impulse response of the components of the real-exchange-rate definition in the model, $R E R:=e P_{Y} / P_{Y}^{*}$ to a $1 \%$ total factor productivity shock in the home country.

\section{[ Figure 2 about here. ]}

Figure 3 considers that of a $1 \%$ home money supply growth shock. The resulting dynamics of the relative cross-country aggregate price deflators are such that they are not so sensitive to technology shocks. By definition then, the dynamics of the real exchange rate must be tracking that of the nominal exchange very well, resulting in a near perfectly positive correlation between the two time series. In standard sticky-price models (see e.g. Chari et al., 2002), the assumption of price stickiness plays a similar, but more obvious, role. However, in our model, this appears to be an equilibrium outcome arising, in part, from the DM anonymity assumption and its resulting restriction of asset and relative pricing dynamics. These figures thus confirm our conjecture in Section 4.

\section{[ Figure 3 about here. ]}

\subsection{Inspecting the Mechanism: Baseline with DM Price-taking}

Recall that in Section 4, we provided the explanation of the potential effects of the assumptions of anonymity (and its resulting monetary equilibrium determination) and capital complementarity on relative pricing processes, and therefore equilibrium exchange rates. In this section, we revisit our explanations, by conducting some experiments to identify the role of each of these mechanisms.

Table 4 summarizes these experiments, which are: (i) Benchmark $(\kappa>0, \varpi>1)$ : the baseline monetary equilibrium with DM price-taking assumption; (ii) Limit $(\kappa=0, \varpi>$ 1): No anonymity (or equivalently a two-sector traded/non-traded goods real business cycle equilibrium); (iii) Limit ( $\kappa>0, \varpi=1)$ : case (i) without DM capital complementarity; and (iv) Limit $(\kappa=0, \varpi=1)$ : No-anonymity version of (iii).

Consider the limit economy (ii) with pure credit trades $(\kappa=0)$ in the DM. This case shuts down completely the role of anonymity and hence monetary friction. This limit economy also identifies a remainder structure: a (separable-utility) version of a standard two-sector realbusiness-cycle model with traded and nontraded goods, and a single capital stock linking both sectors. Moving from column (ii) to column (i), or alternatively from column (iv) to (iii), in Table 4, we can account for the role of anonymity in the DM in explaining real exchange rate (RER) excess volatility. Doing so, we can see that having anonymity in the DM can still qualitatively account for the RER stylized fact: That the RER is more volatile than U.S. output. However, now it can only account for about 47\% (when DM capital complementarity 
Table 4: Inspecting the mechanism: Frictions

\begin{tabular}{|c|c|c|c|c|c|}
\hline & Data & $\begin{array}{c}\text { (i) } \\
\text { Benchmark } \\
(\kappa>0, \varpi>1)\end{array}$ & $\begin{array}{c}\text { (ii) } \\
\text { No Anonymity } \\
(\kappa=0, \varpi>1)\end{array}$ & $\begin{array}{c}\text { (iii) } \\
\text { No DM capital } \\
(\kappa>0, \varpi=1)\end{array}$ & $\begin{array}{l}\text { (iv) } \\
\text { No Anonymity } \\
\text { and } \\
\text { No DM capital } \\
(\kappa=0, \varpi=1)\end{array}$ \\
\hline \multicolumn{6}{|l|}{ Standard deviation: } \\
\hline Nominal E.R., $e$ & 3.34 & 4.8248 & 5.7938 & 5.8543 & 6.5427 \\
\hline Real E.R., $R E R$ & 3.36 & 2.3354 & 1.5637 & 2.2164 & 1.5251 \\
\hline Consumption, $C$ & 0.72 & 0.6107 & 0.6587 & 0.6016 & 0.6671 \\
\hline Investment, $I$ & 2.70 & 1.8210 & 1.7443 & 2.3313 & 2.2250 \\
\hline Hours, $H_{\text {tot }}$ & 0.83 & 0.4608 & 0.4862 & 0.4532 & 0.5661 \\
\hline Price ratio, $P_{Y}^{*} / P_{Y}$ & 0.71 & 2.5203 & 4.3608 & 3.6720 & 5.1251 \\
\hline \multicolumn{6}{|l|}{ Autocorrelation: } \\
\hline Nominal E.R., $e$ & 0.83 & 0.6643 & 0.6687 & 0.6643 & 0.6688 \\
\hline Real E.R., RER & 0.84 & 0.6551 & 0.6486 & 0.6504 & 0.6424 \\
\hline Consumption, $C$ & 0.87 & 0.8190 & 0.8143 & 0.8794 & 0.8215 \\
\hline Investment, $I$ & 0.90 & 0.7832 & 0.8403 & 0.8284 & 0.8702 \\
\hline Hours, $H_{\text {tot }}$ & 0.94 & 0.9175 & 0.9196 & 0.9152 & 0.9152 \\
\hline Output, $Y$ & 0.89 & 0.7932 & 0.8258 & 0.8280 & 0.8338 \\
\hline Price ratio, $P_{Y}^{*} / P_{Y}$ & 0.87 & 0.6744 & 0.6814 & 0.6733 & 0.6791 \\
\hline
\end{tabular}

is present: (ii) to (i)), or about $45 \%$ (when DM complementarity is not present: (iii) to (iv)), of this excess volatility in the data.

Note that columns (ii) and (i) of Table 4 represent economies with capital linking both the DM (nontraded good sector) and the CM (traded good sector). We would also like to see what additional contribution the assumption of capital complementarity in the DM (nontraded good sector) plays in generating the excess-volatility stylized fact of the RER in the models. This exercise is shown in Columns (iii) and (iv) of Table 4.

Comparing columns (iii) and (i), the contribution of capital complementarity in the DM to the excess volatility in the RER is positive. However, in contrast to the contribution of DM anonymity alone, the contribution is smaller. Similarly, suppose that there is no anonymity; then we consider moving from economies (iv) to (ii) in Table 4. Again, having capital complementarity help account for more excess volatility in the RER, but that contribution is not as large as anonymity per se.

In summary, the assumption of anonymity or DM capital complementarity per se, can contribute to account for additional excess volatility of the RER. The marginal contribution of the anonymity assumption per se is bigger than that of the DM capital complementarity assumption. However, when both assumptions are present, we can account for the excess volatility in the RER even better. In this exercise, we have also verified that the informational friction of anonymity is not only a means of introducing money into models after Lagos and Wright (2005), but they also matter for stochastic equilibrium relative pricing dynamics. In our case of the DM price-taking protocol, our $g$ function indeed is able to produce what we conjectured from analyzing the model's SME conditions in Section 4. 
We now turn our attention to the autocorrelation (persistence) properties of the RER in Table 4. Consider first, moving from an economy with no anonymity in DM trades (column (ii)) to an economy with some anonymity (Column (i)), the real exchange rate's first-order autocorrelation improves from 0.6486 to 0.6551 , i.e. approximately $1 \%$. Second, moving from an economy with no DM capital complementarity (Column (iii)) to an economy with some complementarity (column (i)), the real exchange rate's first-order autocorrelation improves from 0.6504 to 0.6551 , i.e. by about $0.7 \%$. These quantitatively calibrated experiments show that both anonymity (and therefore its implied market incompleteness or lack of consumption smoothing) and DM capital complementarity contribute to making the real exchange rate more persistent, albeit the contribution is quite small.

\subsection{Alternative DM Nash bargaining model}

For completeness, we also consider Nash bargaining, originally used in Lagos and Wright (2005), as an alternative DM pricing mechanism. The interpretation now is that agents are bilaterally matched in a random fashion with $\sigma \kappa$ being the joint probability of the event that an agent meets another agent who is able to produce the special good she wants, and, that trade is anonymous. With identical probability $\sigma \kappa$ an agent meets another who wishes to buy the special good she can produce. Alternatively, similar events (agent as buyer or as seller) which are monitored, each occur with probability $\sigma(1-\kappa)$. Thus with probability $1-2 \sigma$ an agent leaves the DM with no exchange. ${ }^{18}$

We calibrate this alternative model to the same empirical targets as in the benchmark model. However, we now have an additional parameter $\theta$ representing the common bargaining strength of the buyer in both monetary and credit exchanges. Following Aruoba et al. (2011), we calibrate this parameter, jointly with the others, to match a steady state aggregate pricing markup of around $33 \%$.

The business cycle dynamics of this alternative model are reported in Table 5. Qualitatively, this version of the model is able to account for the observed excess volatility and persistence in the nominal and real exchange rates. However, these come at a cost of a counterfactually volatile consumption and investment process (in excess of output volatility). Also, the real and nominal exchange rates are counterfactually and negatively correlated. ${ }^{19}$

Finally, neither of the models we have considered come close to addressing the BackusSmith consumption real exchange rate correlation anomaly. In the data, the correlation is often negative, whereas in most models it is almost or is perfectly and positively correlated. This remains a puzzle with respect to the class of models we considered. As shown in Backus and Smith (1993), when the aggregate price levels comprise traded and nontraded goods, the

\footnotetext{
${ }^{18}$ The characterization of a monetary equilibrium under Nash bargaining is quite standard (see e.g. Aruoba et al., 2011; Aruoba, 2010) and can be found in a separate appendix to this paper.

${ }^{19}$ We show and discuss in a supplementary appendix why this may be the case in this model.
} 
Table 5: Data and alternative equilibrium statistics

\begin{tabular}{lccc}
\hline \hline & Data & DM Price Taking & DM Nash Bargaining \\
\hline Standard deviation: & & & \\
Nominal E.R., $e$ & 3.34 & 4.82 & 5.30 \\
Real E.R., RER & 3.36 & 2.34 & 8.64 \\
Consumption, $C$ & 0.72 & 0.61 & 0.85 \\
Investment, $I$ & 2.70 & 1.82 & 12.54 \\
Hours, $H_{\text {tot }}$ & 0.83 & 0.46 & 1.15 \\
Autocorrelation: & & & \\
Nominal E.R., $e$ & 0.83 & 0.66 & 0.65 \\
Real E.R., RER & 0.84 & 0.66 & 0.65 \\
Consumption, $C$ & 0.87 & 0.82 & 0.43 \\
Investment, $I$ & 0.90 & 0.78 & 0.44 \\
Hours, $H_{\text {tot }}$ & 0.94 & 0.92 & 0.52 \\
Output, $Y$ & 0.89 & 0.79 & \\
& & & -0.96 \\
Contemporaneous correlation: & & & -0.73 \\
(RER, $)$ & 0.99 & 0.99 & 0.99 \\
$(R E R, N X)$ & 0.14 & 0.17 & \\
$\left(R E R, C^{*} / C\right)$ & -0.35 & 0.91 & \\
\hline \hline
\end{tabular}

perfect correlation between RER and relative consumption across countries, $C^{*} / C$, can be broken. In our benchmark calibrated model with such a feature as well, this correlation is a mere 0.9130 (see Table 5). In other words, the model is still unable to account for the bulk of the observed correlation $(-0.35)$ between RER and $C^{*} / C$ in the data.

\section{Conclusion}

In this paper, we examined whether a flexible price, two-country, search theoretic model of money is able to account for the empirical regularities observed in U.S. real and nominal exchange rate dynamics. We proposed a two-country version of Aruoba et al. (2011) where international trade and asset flows occur in the model's Walrasian centralized markets.

There are two key mechanisms at work in this model that help amplify and propagate international business cycle shocks. The first mechanism is anonymity. This friction induces asset market incompleteness in the sense that individuals are unable to fully insure against their stochastic trading opportunities in the decentralized markets (DM). The second mechanism is the notion of capital complementarity. The latter mechanism provides for an additional return on capital which places additional restriction on the equilibrium asset pricing relations with respect to money and capital.

We show that the relative pricing dynamics of the baseline model behave in such a way that cross-country aggregate relative prices are non-volatile and persistent. This contributes to the excess volatility and persistence in the real and nominal exchange rate. Without requiring exogenous price-stickiness, we are also able to rationalize near perfect positive correlation between the real and nominal exchange rate. Thus monetary friction, in the sense of Lagos and Wright (2005), is more than just a vehicle for a theoretical foundation of money. In a 
stochastic two-country environment, it restricts asset pricing relations such that the model is able to account for the stylized facts on real and nominal exchange rate fluctuations.

Future quantitative theory in this direction should consider deeper foundations of the coexistence of multiple currencies and assets. This is currently a weakness in our model. Providing a theory that simultaneously rationalizes the coexistence of means of payments, and, that accounts for international monetary business cycle facts would be an interesting open challenge.

Pere Gomis-Porqueras. Department of Economics, Monash University, Victoria 3145, Australia

Tiмотну KАм. Research School of Economics, The Australian National University, ACT 0200, Australia

Junsang Lee. Korea Development Institute, P.O.Box 113, Hoegiro 49, Dongdaemun-gu, Seoul, 130-868, Republic of Korea

\section{A Data}

We focus on quarterly data spanning from Quarter 1 of 1975 to Quarter 4 of 2004. Following Heathcote and Perri (2002) we measure employment $H_{t o t}$ using the OECD MEI Civilian Employment Index. We obtain measures of the U.S. nominal and real effective exchange rates, as proxies for $e$ and $R E R$, respectively, from the International Monetary Fund's International Financial Statistics (IFS). We measure aggregate private consumption $(C)$, investment $(I)$ and net exports $(N X)$ from the OECD Outlook Quarterly database. Real output is just a sum of these components.

\section{References}

Alessandria, G., "Consumer Search, Price Dispersion, And International Relative Price Fluctuations," International Economic Review 50 (08 2009), 803-829.

Alvarez, F. And M. Veracierto, "Labor-Market Policies in an Equilibrium Search Model," in NBER Macroeconomics Annual 1999, Volume 14NBER Chapters (National Bureau of Economic Research, 2000), 265-316.

AruobA, S. B., "Money, Search and Business Cycles," Forthcoming International Economic Review, University of Maryland, April 2010.

Aruoba, S. B., C. J. Waller And R. Wright, "Money and Capital," Journal of Monetary Economics 58 (2011), 98-116. 
Aruoba, S. B. And R. Wright, "Search, Money and Capital: A Neoclassical Dichotomy," Journal of Money, Credit and Banking 35 (2003), 1086-1105.

Backus, D. K., P. J. Kehoe and F. E. Kydland, "Dynamics of the Trade Balance and the Terms of Trade: The J-Curve?," American Economic Review 84 (March 1994), 84-103.

Backus, D. K. And G. W. Smith, "Consumption and Real Exchange Rates in Dynamic Economies with Non-traded Goods," Journal of International Economics 35 (August 1993), 297-316.

Burdett, K. And K. L. Judd, "Equilibrium Price Dispersion," Econometrica 51 (July 1983), $955-69$.

Chari, V. V., P. J. Kehoe and E. R. McGrattan, "Can Sticky Price Models Generate Volatile and Persistent Real Exchange Rates?," Review of Economic Studies 69 (July 2002), $533-63$.

Dornbusch, R., "Expectations and Exchange Rate Dynamics," Journal of Political Economy 84 (1976), 1161-1176.

Duffie, D., J. Geanakoplos, A. Mas-Colell and A. Mclennan, "Stationary Markov Equilibria," Econometrica 62 (July 1994), 745-81.

Head, A. And S. Shi, "A Fundamental Theory of Exchange Rates and Direct Currency Trades," Journal of Monetary Economics 50 (October 2003), 1555-1591.

Heathcote, J. And F. Perri, "Financial Autarky and International Business Cycles," Journal of Monetary Economics 49 (April 2002), 601-627.

Lagos, R. And R. Wright, "A Unified Framework for Monetary Theory and Policy Analysis," Staff Report 346, Federal Reserve Bank of Minneapolis, 2004.

—_, "A Unified Framework for Monetary Theory and Policy Analysis," Journal of Political Economy 113 (June 2005), 463-484.

Lester, B., A. Postlewaite and R. Wright, "Information, Liquidity and Asset Prices," PIER Working Paper Archive 08-039, Penn Institute for Economic Research, Department of Economics, University of Pennsylvania, October 2008.

Li, Y. And G. Rocheteau, "Liquidity Constraints," Technical Report, U.C. Irvine, 2009.

Lucas, R. J. And E. C. Prescott, "Equilibrium Search and Unemployment," Journal of Economic Theory 7 (February 1974), 188-209. 
Rocheteau, G. and R. Wright, "Money in Search Equilibrium, in Competitive Equilibrium, and in Competitive Search Equilibrium," Econometrica 73 (01 2005), 175-202.

Schlagenhauf, D. E. And J. Wrase, "Exchange Rate Dynamics and International Effects of Monetary Shocks in Monetary Equilibrium Models," Journal of International Money and Finance 14 (1995), 155-177.

Steinsson, J., "The Dynamic Behavior of the Real Exchange Rate in Sticky Price Models," American Economic Review 98 (March 2008), 519-33.

Stockman, A. C. And L. L. Tesar, "Tastes and Technology in a Two-Country Model of the Business Cycle: Explaining International Comovements," American Economic Review 85 (March 1995), 168-85.

Waller, C. J., "Random Matching and Money in the Neoclassical Growth Model: Some Analytical Results," Macroeconomic Dynamics forthcoming (2010). 


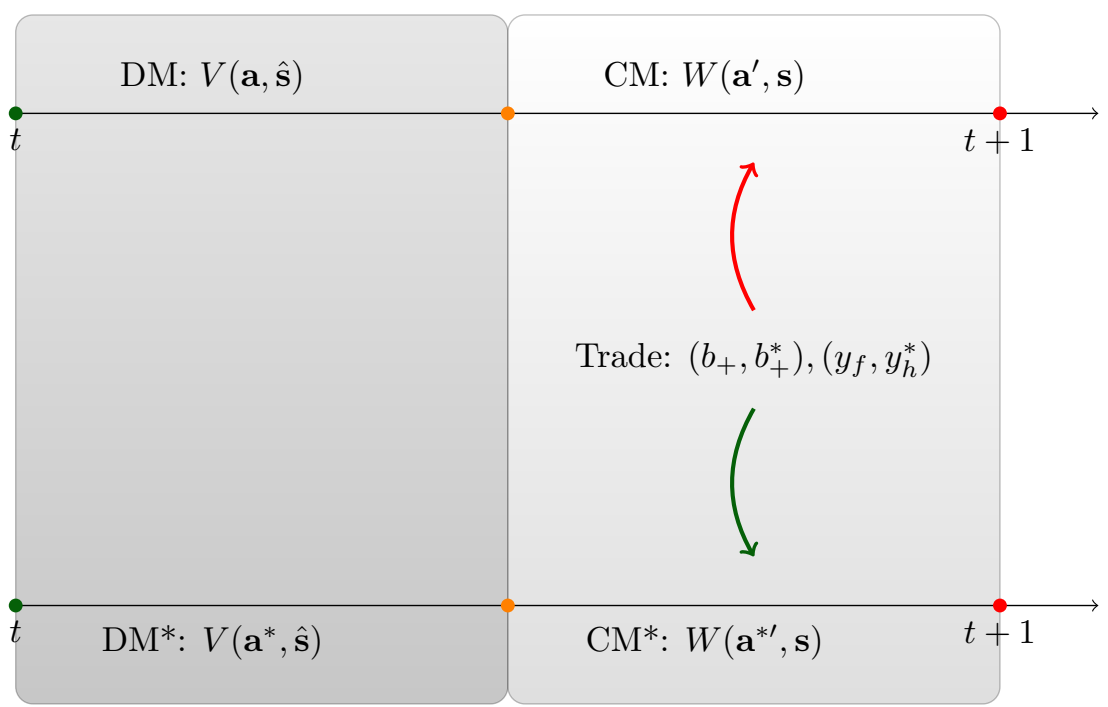

Figure 1: Timing 
Figure 2: DM Price taking. Real and nominal exchange rates versus relative aggregate prices: $1 \%$ Home TFP increase $z$.

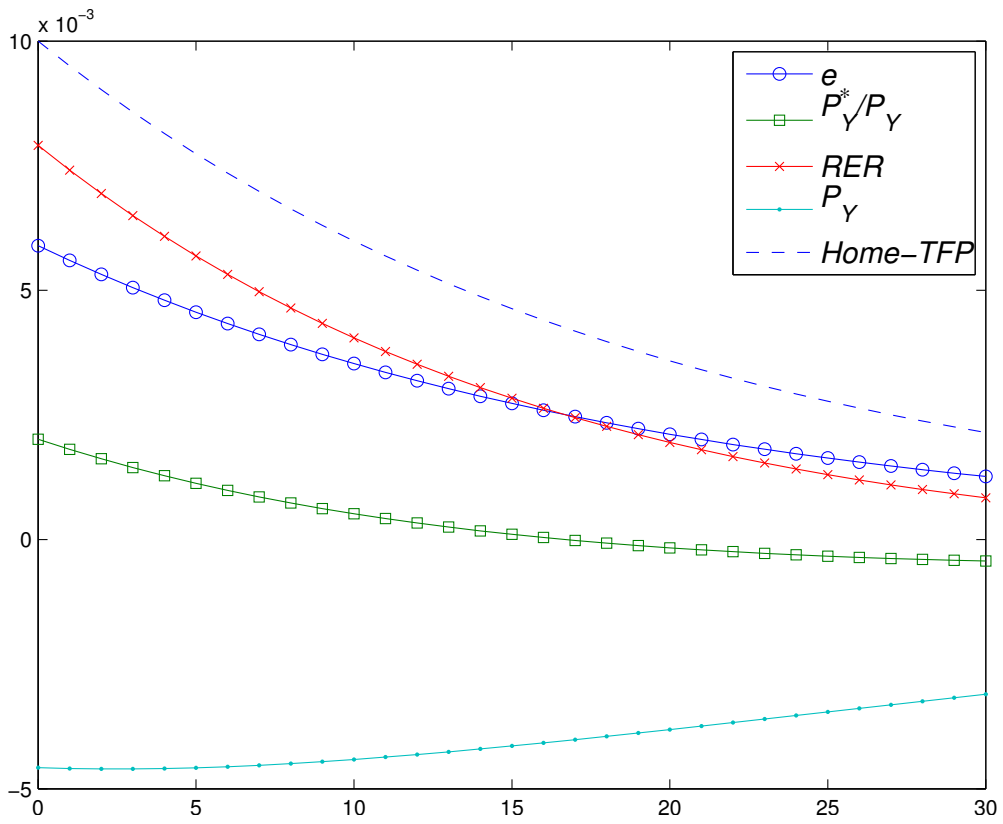

Figure 3: DM Price taking. Real and nominal exchange rates versus relative aggregate prices: $1 \%$ Home money supply growth increase, $\psi$.

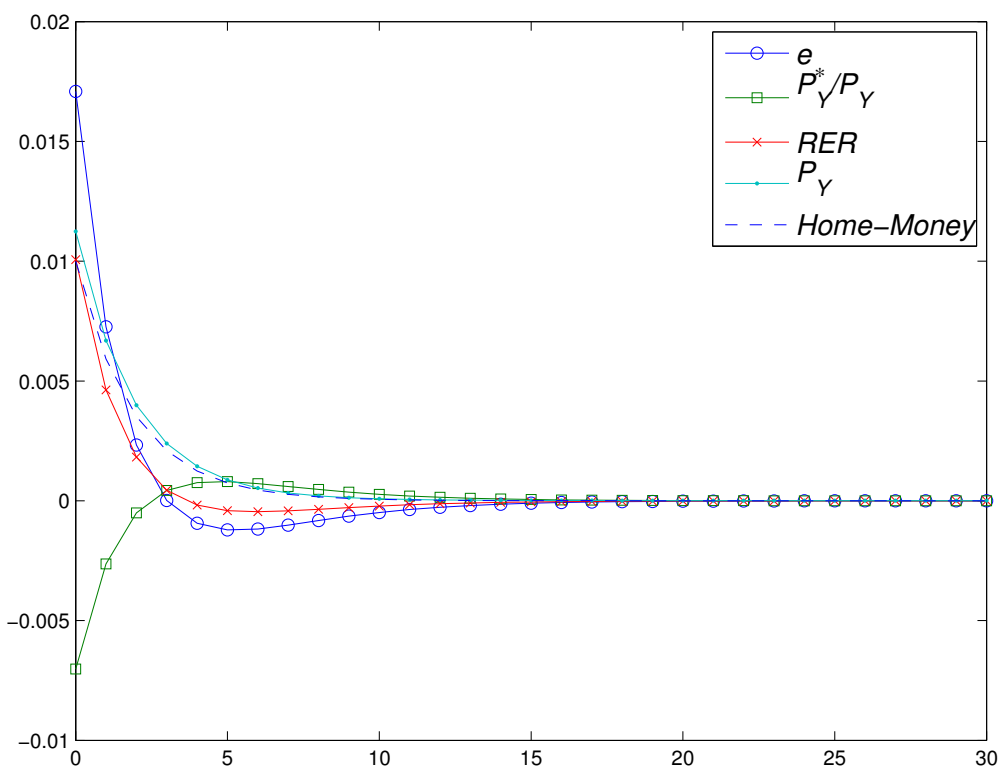

